Annales Geophysicae (2003) 21: 1691-1707 (C) European Geosciences Union 2003

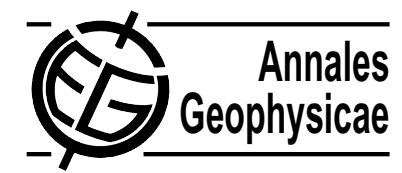

\title{
Corotation-driven magnetosphere-ionosphere coupling currents in Saturn's magnetosphere and their relation to the auroras
}

\author{
S. W. H. Cowley and E. J. Bunce \\ Department of Physics and Astronomy, University of Leicester, Leicester LE1 7RH, UK
}

Received: 5 November 2002 - Revised: 6 March 2003 - Accepted: 18 March 2003

\begin{abstract}
We calculate the latitude profile of the equatorward-directed ionospheric Pedersen currents that are driven in Saturn's ionosphere by partial corotation of the magnetospheric plasma. The calculation incorporates the flattened figure of the planet, a model of Saturn's magnetic field derived from spacecraft flyby data, and angular velocity models derived from Voyager plasma data. We also employ an effective height-integrated ionospheric Pedersen conductivity of 1 mho, suggested by a related analysis of Voyager magnetic field data. The Voyager plasma data suggest that on the largest spatial scales, the plasma angular velocity declines from near-rigid corotation with the planet in the inner magnetosphere, to values of about half of rigid corotation at the outer boundary of the region considered. The latter extends to $\sim 15-20$ Saturn radii $\left(R_{S}\right)$ in the equatorial plane, mapping along magnetic field lines to $\sim 15^{\circ}$ co-latitude in the ionosphere. We find in this case that the ionospheric Pedersen current peaks near the poleward (outer) boundary of this region, and falls toward zero over $\sim 5^{\circ}-10^{\circ}$ equatorward of the boundary as the plasma approaches rigid corotation. The peak current near the poleward boundary, integrated in azimuth, is $\sim 6 \mathrm{MA}$. The field-aligned current required for continuity is directed out of the ionosphere into the magnetosphere essentially throughout the region, with the current density peaking at $\sim 10 \mathrm{nA} \mathrm{m}^{-2}$ at $\sim 20^{\circ}$ co-latitude. We estimate that such current densities are well below the limit requiring field-aligned acceleration of magnetospheric electrons in Saturn's environment $\left(\sim 70 \mathrm{nA} \mathrm{m}^{-2}\right)$, so that no significant auroral features associated with this ring of upward current is anticipated. The observed ultraviolet auroras at Saturn are also found to occur significantly closer to the pole (at $\sim 10^{\circ}-15^{\circ}$ co-latitude), and show considerable temporal and local time variability, contrary to expectations for corotation-related currents. We thus conclude that Saturn's 'main oval' auroras are not associated with corotationenforcing currents as they are at Jupiter, but instead are most probably associated with coupling to the solar wind as at
\end{abstract}

Correspondence to: S. W. H. Cowley (swhc1@ion.le.ac.uk)
Earth. At the same time, the Voyager flow observations also suggest the presence of radially localized 'dips' in the plasma angular velocity associated with the moons Dione and Rhea, which are $\sim 1-2 R_{S}$ in radial extent in the equatorial plane. The presence of such small-scale flow features, assumed to be azimuthally extended, results in localized several-MA enhancements in the ionospheric Pedersen current, and narrow bi-polar signatures in the field-aligned currents which peak at values an order of magnitude larger than those associated with the large-scale currents. Narrow auroral rings (or partial rings) $\sim 0.25^{\circ}$ co-latitude wide with intensities $\sim 1$ kiloRayleigh may be formed in the regions of upward field-aligned current under favourable circumstances, located at co-latitudes between $\sim 17^{\circ}$ and $\sim 20^{\circ}$ in the north, and $\sim 19^{\circ}$ and $\sim 22^{\circ}$ in the south.

Key words. Magnetospheric physics (current systems; magnetosphere-ionosphere interactions; planetary magnetospheres)

\section{Introduction}

The first indications that auroral emissions occur in Saturn's polar regions were obtained by the ultraviolet (UV) photometer on the Pioneer-11 spacecraft during the flyby in 1979 (Judge et al., 1980), and remotely from observations by the IUE spacecraft (Clarke et al., 1981; McGrath and Clarke, 1992). The first unambiguous detections were made by the UVS instruments on the two Voyager spacecraft during the flybys in 1980 and 1981 (Broadfoot et al., 1981; Sandel and Broadfoot, 1981; Sandel et al., 1982; Shemansky and Ajello, 1983). These latter observations indicated that the UV auroras are excited by $\sim 10 \mathrm{keV}$ electron excitation of molecular hydrogen, are variable in intensity over a range from a few $\mathrm{kR}$ to $\sim 100 \mathrm{kR}$ of total $\mathrm{H}_{2}$ emission on time scales from tens of minutes to hours, take the form of circumpolar bands around both northern and southern poles of the planet, which are essentially continuous in local time, and are confined to 


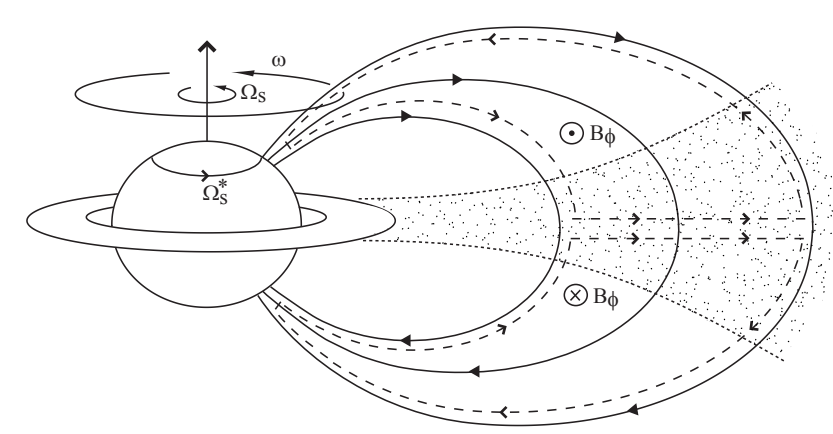

Fig. 1. Sketch of a meridian cross section through Saturn's quasiaxisymmetric inner and middle magnetosphere, extending to distances of $\sim 15-20 R_{S}$ in the equatorial plane. The arrowed solid lines indicate magnetic field lines, which are modestly distended outward from the planet by azimuthal currents (the 'ring-current') flowing in the near-equatorial plasma. The rotating plasma is shown by the dotted region, consisting mainly of protons and oxygen ions and associated electrons, which derive from water ice originating from ring grains and moon surfaces. Three angular velocities are indicated. These are the angular velocity of a particular shell of field lines $\omega$, the angular velocity of the planet $\Omega_{S}$, and the angular velocity of the neutral upper atmosphere in the Pedersen layer of the ionosphere, $\Omega_{S}^{*}$. The value of $\Omega_{S}^{*}$ is expected to lie between $\omega$ and $\Omega_{S}$ because of the frictional torque on the atmosphere due to ion-neutral collisions. The oppositely-directed frictional torque on the magnetospheric flux tubes is communicated to the equatorial plasma by the current system indicated by the arrowed dashed lines, shown here for the case of sub-corotation of the plasma (i.e. $\omega<\Omega_{S}$ ). This current system bends the field lines out of meridian planes into a 'lagging' configuration, associated with the azimuthal field components $B_{\varphi}$ shown.

co-latitudes less than $\sim 12^{\circ}$ from each pole. The most precise determination of the latitude distribution, obtained by Voyager-1 near closest approach, placed the Southern Hemisphere aurora near dusk between co-latitudes of $\sim 9.5^{\circ}$ and $\sim 12^{\circ}$ with respect to the southern pole (Sandel and Broadfoot, 1981). The intensity at fixed local time was also found to be strongly modulated during each planetary rotation, approximately in phase with a similar modulation of Saturn kilometric radiation (SKR) (Gurnett et al., 1981; Sandel and Broadfoot, 1981; Sandel et al., 1982; Kaiser et al., 1984). Since the SKR emissions were found to be positively correlated with the solar wind dynamic pressure at Saturn (Desch, 1982; Desch and Rucker, 1983), the inference has also been drawn of a possible connection between the UV auroras and solar wind-magnetosphere coupling processes. This suggestion is supported by estimates of $\sim 11^{\circ}-15^{\circ}$ for the colatitude of the boundary of field lines mapping into Saturn's tail, and thus to the magnetopause vicinity, determined from Voyager-1 tail magnetic field data (Ness et al., 1981).

More recently, images of Saturn's UV aurora have been obtained by the Hubble Space Telescope (HST). The first such image was presented by Gérard et al. (1995), which suggested a ring of emission around the north pole at $\sim 10^{\circ}$ co-latitude, with an intensity of $\sim 150 \mathrm{kR}$ of total $\mathrm{H}_{2}$ emis- sion. Further HST images have been presented by Trauger et al. (1998). Emissions above a $\sim 5 \mathrm{kR}$ threshold were again observed over a wide range of local times in the northern dayside hemisphere, but were found to be strongly peaked between 07:00-09:00 LT in the morning sector, in the colatitude range $13^{\circ}-15^{\circ}$. This location appears to be consistent with the principal source region of right-hand polarised SKR radio emissions (Kaiser and Desch, 1982; Lecacheux and Genova, 1983). However, as for the Voyager observations, the auroras again show considerable variation in intensity between images which are well separated in time, varying from no emission at all above the few-kR threshold, to emissions peaking (in the morning sector) at $\sim 100 \mathrm{kR}$.

The main auroral emissions at Jupiter also consist of bands around both poles of the planet (e.g. Satoh et al., 1996; Prangé et al., 1998; Clarke et al., 1998; Vasavada et al., 1999), though in this case they appear to be considerably less variable in both UT and local time (Grodent et al., 2003). However, these emissions, located at $\sim 15^{\circ}$ magnetic colatitude, map magnetically to middle magnetosphere field lines at equatorial distances beyond $\sim 20 R_{J}$ (Gérard et al., 1994; Prangé et al., 1998; Clarke et al., 1998), and have been suggested to be associated with the field-aligned currents of a current system which enforces partial corotation of plasma moving outward from the Io torus (Bunce and Cowley, 2001; Hill, 2001; Southwood and Kivelson, 2001). Cowley and Bunce (2001) have set up a quantitative empirical model of this current system, and have shown using Knight's (1973) kinetic theory that the precipitated energy flux of downward-accelerated auroral electrons associated with the region of upward current is sufficient to produce the $\sim 100 \mathrm{kR}$ to $\sim 1 \mathrm{MR}$ auroras observed.

The basic physical processes occurring in the middle regions of Saturn's magnetosphere (as opposed, for example, to the outer regions where solar wind processes may become important) are believed to be essentially similar to those at Jupiter, if on a lesser scale, involving the pick-up and radial transport of plasma originating from ring particles and moons in a corotation-dominated flow (e.g. Richardson, 1992; Richardson et al., 1998). Indeed, observations from the Voyager spacecraft have shown that as a result of these processes the plasma in Saturn's magnetosphere subcorotates relative to the planet (Richardson, 1986; Richardson and Sittler, 1990). Corotation-enforcement currents, as first discussed by Hill (1979) and Vasyliunas (1983) in the jovian context, must therefore also flow at Saturn. Their form is illustrated by the dashed lines in Fig. 1, as will be discussed further below. Given their proposed significance at Jupiter, the question is thus raised concerning their magnitude and distribution at Saturn, and possible relationship to the observed auroral morphology. In this paper we examine this issue by making a quantitative calculation of the corotation-enforcement currents at Saturn based on Pioneer and Voyager observations, from which we then assess the auroral consequences. 


\section{Theoretical model}

The calculation of the magnetosphere-ionosphere coupling currents that results from sub-corotation of the magnetospheric plasma is straightforward, in principle. The angular velocity of the plasma observed in the magnetosphere is mapped along magnetic field lines into the ionosphere, where it determines the electric field in the rest frame of the neutral atmosphere, and hence, the horizontal current flowing in the ionosphere. The field-aligned current flowing between the ionosphere and magnetosphere is then determined from the divergence of the latter current, from which the potential for field-aligned electron acceleration and enhanced auroral emission associated with regions of upward-directed fieldaligned current can be assessed. In order to accomplish this programme, however, a number of issues must be addressed. First, we need a magnetic model to map field lines from the points in the magnetosphere where the plasma angular velocity is measured into the polar ionosphere. Second, we need to calculate the horizontal ionospheric current intensity from the height-integrated conductivity and the $-\boldsymbol{V} \times \boldsymbol{B}$ electric field, evaluated in the rest frame of the neutral atmosphere. Third, we need to calculate the field-aligned current density and associated auroral parameters. These topics will now be addressed in turn.

\subsection{Magnetic mapping to the ionosphere}

Spacecraft flyby measurements have shown that Saturn's internal magnetic field is closely symmetric about the spin axis of the planet, with a magnetic axis that lies well within $\sim 1^{\circ}$ of the spin axis, such that it can be modelled as the sum of axial dipole, quadrupole and octupole terms (Connerney et al., 1982, 1984; Davis and Smith, 1990). In the magnetosphere, the internal field is inflated by the presence of currents flowing in the equatorial plasma, but these effects can be modelled by including a ring current which is also axisymmetric in a first approximation (Connerney et al., 1983; Bunce and Cowley, 2003). Here, therefore, in order to map measured plasma angular velocities from the corotationdominated regions of Saturn's magnetosphere into the ionosphere, we have assumed that the magnetic field as a whole is axisymmetric about the spin axis of the planet. Axisymmetry is broken at large distances, of course, where the effects of the magnetopause and tail currents become important. However, the approximation employed here should generally be sufficient to distances $\sim 15-20 R_{S}$ in the equatorial plane (depending, for example, on the dynamic pressure of the solar wind and the degree of extension of the magnetosphere), compared with subsolar magnetopause distances of $\sim 17-24 R_{S}$ (Behannon et al., 1983; Maurice and Engel, 1995). As we will find below, radial distances of $15-20 R_{S}$ in the equatorial plane map to the ionosphere in the co-latitude range $\sim 13^{\circ}-15^{\circ}$ in the Northern Hemisphere and $\sim 14^{\circ}-17^{\circ}$ in the south (with respect to the southern pole). Here, $R_{S}$ is the conventional Saturn radius, equal to $60330 \mathrm{~km}$.
Assuming, then, an axisymmetric field about the planet's spin axis, the poloidal field can be described by a flux function $F(r, \theta)$, related to the field components by $\boldsymbol{B}=$ $(1 / r \sin \theta) \nabla F \times \hat{\boldsymbol{\varphi}}$. Here, we employ spherical polar coordinates, with $r$ the distance from the centre of the planet, $\theta$ the co-latitude angle measured from the north pole, and $\varphi$ the azimuthal angle. The surface $F=$ constant defines an azimuthally-symmetric shell of field lines passing from the northern ionosphere, through the equator, to the southern ionosphere. In order to map from some magnetospheric observation point to the ionosphere, therefore, we simply compute the value of the flux function $F$ at that point, and solve $F=F_{i}$, where $F_{i}$ is the flux function in the ionosphere. The flux function for the internal field is

$$
\begin{aligned}
& F_{\text {int }}(r, \theta)=R_{S}^{2} \sin ^{2} \theta\left[g_{1}^{0}\left(\frac{R_{S}}{r}\right)\right. \\
& \left.\quad+\frac{3}{2} g_{2}^{0} \cos \theta\left(\frac{R_{S}}{r}\right)^{2}+\frac{1}{2} g_{3}^{0}\left(5 \cos ^{2} \theta-1\right)\left(\frac{R_{S}}{r}\right)^{3}\right]
\end{aligned}
$$

where the $g$ 's are the dipole, quadrupole, and octupole coefficients. The absolute value of $F$ has been fixed by taking $F=0$ on the magnetic axis. In this paper we use the SPV model coefficients of Davis and Smith (1990), obtained from a fit to magnetic data from all three flyby data sets. The values are $g_{1}^{0}=21160, g_{2}^{0}=1560$, and $g_{3}^{0}=2320 \mathrm{nT}$ for a conventional Saturn radius of $R_{S}=60330 \mathrm{~km}$. (We note that this value for Saturn's radius, roughly the radial distance of the 1 bar level at the equator, has been employed in most previous modelling and data analysis efforts, and, for simplicity, has consequently also been adopted here. However, we note that the value of the radial distance of the 1 bar level at the equator currently recommended by the IAU is $60268 \mathrm{~km}$.)

In the magnetosphere the flux function due to the ring current must be added to $F_{i n t}$, and since we will employ the angular velocities measured during the two Voyager flybys, we also employ the ring current model derived from fits to the Voyager magnetic field data by Connerney et al. (1983). In this model the azimuthal current is cylindrically symmetric about the spin axis of the planet, and flows in the region between cylindrical radial distances $a_{1}=8 R_{S}$ and $a_{2}=15.5 R_{S}$, and to a distance $D=3 R_{S}$ on either side of the equator. Within this region the azimuthal current density falls inversely as the cylindrical radial distance from the axis. Outside the region the current is zero. The flux function for this current distribution, which we term the 'Connerney, Acuña, and Ness' or 'CAN' model, is obtained numerically by evaluation of exact integral formulas given by Connerney et al. (1981) (see also Edwards et al., 2001). Employing cylindrical co-ordinates $(\rho, z)$, we have for $|z| \leq D$

$$
\begin{aligned}
& F_{C A N}^{i}(\rho, z)=\left(\mu_{o} I_{o}\right) \rho \int_{0}^{\infty} \frac{d \lambda}{\lambda^{2}} J_{1}(\rho \lambda) \times \\
& \quad \times\left[J_{0}\left(a_{1} \lambda\right)-J_{0}\left(a_{2} \lambda\right)\right]\left(1-e^{-D \lambda} \cosh (z \lambda)\right),
\end{aligned}
$$



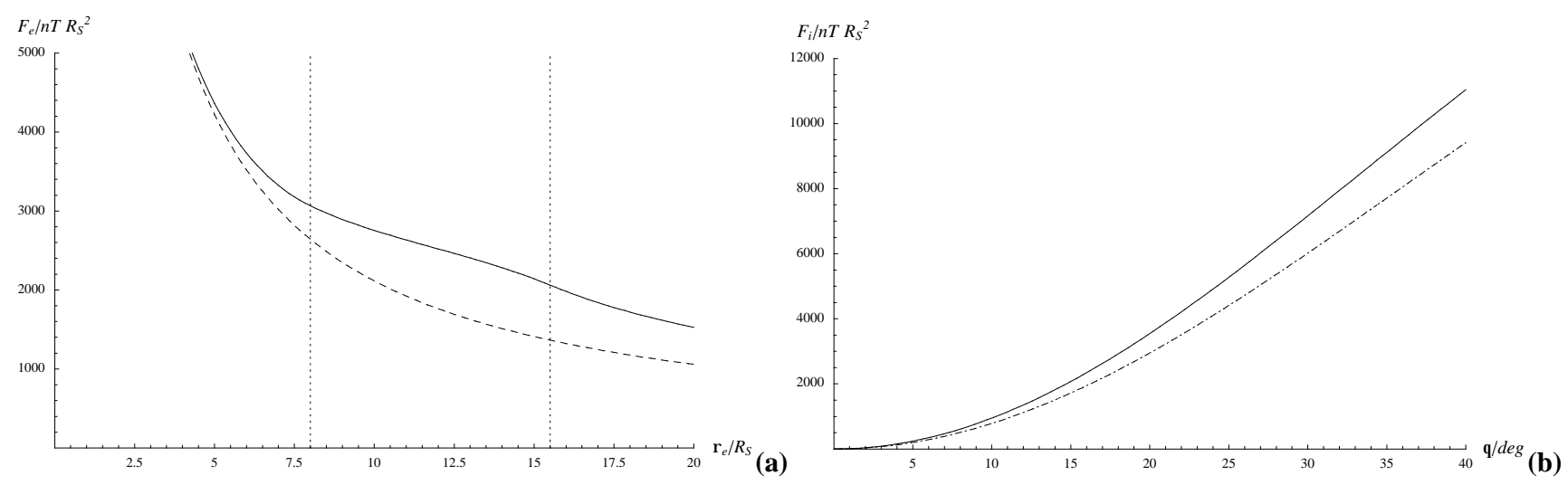

Fig. 2. (a) Plot of the magnetic field flux function in the equatorial plane, $F_{e}$, versus equatorial distance from the planet $\rho_{e}$. The units of the flux function are $\mathrm{nT} R_{S}^{2}$, while the distance is normalised to the conventional Saturn radius $R_{S}(60330 \mathrm{~km})$. The dashed line corresponds to the internal field of the planet alone given by Eq. (1) (essentially just the dipole term over the range shown), while the solid line also includes the effect of the Connerney et al. (1983) ring current. The vertical dotted lines show the radial range within which the ring current flows $\left(8-15.5 R_{S}\right)$. (b) Corresponding plot of the flux function in the ionosphere, $F_{i}\left(\mathrm{nT} R_{S}^{2}\right.$ ), versus co-latitude $\theta$, obtained using the internal field alone. The solid line shows results for the Northern Hemisphere, while the dot-dashed line shows results for the Southern Hemisphere (plotted versus co-latitude with respect to the southern pole).

while for $|z| \geq D$

$$
\begin{aligned}
& F_{C A N}^{ \pm}(\rho, z)=\left(\mu_{o} I_{o}\right) \rho \int_{0}^{\infty} \frac{d \lambda}{\lambda^{2}} J_{1}(\rho \lambda) \times \\
& \times\left[J_{0}\left(a_{1} \lambda\right)-J_{0}\left(a_{2} \lambda\right)\right] \sinh (D \lambda) e^{\mp z \lambda}
\end{aligned}
$$

where the upper sign is valid for $z>D$, and the lower sign for $z<-D$. The $J_{n}$ 's are Bessel functions of the first kind. The current intensity parameter determined by Connerney et al. (1983) from a fit to Voyager Saturn flyby magnetometer data is $\mu_{o} I_{o} \approx 60.4 \mathrm{nT}$.

Figure 2a shows the flux function so determined, plotted versus radial distance in the equatorial plane. The dashed line shows the contribution of the internal field only given by Eq. (1), essentially equal to the contribution of the dipole term alone in the range illustrated. The quadrupole term gives no contribution on the equator, and the octupole term is negligible at distances beyond a few $R_{S}$. The solid line then shows the effect of adding the ring current, located in the radial range between the vertical dotted lines, obtained by adding Eqs. (1) and (2) to form the total flux function $F=F_{\text {int }}+F_{C A N}$. This increases the value of $F$ at each point, particularly within the region of the ring current, corresponding to an outward displacement of the field lines due to the plasma current. The field line displacement is given by the distance between the dashed and solid curves at fixed $F$.

In the ionosphere, however, the contribution of the ring current is negligible, so that the ionospheric flux function $F_{i}$ can be determined directly from Eq. (1) once the radial distance $r$ as a function of co-latitude $\theta$ is specified. For many purposes it is sufficient to take a constant value, for example, $r=R_{S}$ in Eq. (1). However, Saturn is the most oblate of all the planets in the solar system, with a polar radius which is less than the equatorial value by $\sim 10 \%$, and this can have a sensible effect on the values of the field components and the flux function in the ionosphere. For example, the north polar field strength given by the SPV model changes from $\sim 55000 \mathrm{nT}$ if we use $r \approx R_{S}$, to $\sim 75000 \mathrm{nT}$ when taking into account the polar flattening. Here, therefore, we have taken the surface representing the conducting layer of the ionosphere to be an ellipse of revolution about the spin axis, with an equatorial radius of $R_{e}$ and a polar radius of $R_{p}$, that is the radial distance of the conducting layer of the ionosphere as a function of co-latitude $\theta$ is taken to be

$R_{i}(\theta)=\frac{R_{e}}{\left(1+\varepsilon \cos ^{2} \theta\right)^{1 / 2}}$ where $\varepsilon=\left(\frac{R_{e}}{R_{p}}\right)^{2}-1$.

We have taken the Pedersen conducting layer of the ionosphere to lie $1000 \mathrm{~km}$ above the 1 bar reference spheroid of the planet, corresponding to the altitude where the ionneutral collision frequency is comparable to the ion gyrofrequency, such that the ions have maximum mobility in the direction of the electric field (e.g. Connerney et al., 1983; Atreya et al., 1984; Moses and Bass, 2000). Following the radial distances given by Trauger et al. (1998), we have thus used $R_{e}=61268 \mathrm{~km} \approx 1.02 R_{S}$ and $R_{p}=55364 \mathrm{~km}$ $\approx 0.92 R_{S}$, such that $\varepsilon \approx 0.22$. Investigation has shown, however, that our results are not sensitive to variations of several hundred $\mathrm{km}$ about these values. Substituting $r=R_{i}(\theta)$ from Eq. (3a) with these values into Eq. (1) then defines the flux function in the ionosphere. This is shown versus colatitude $\theta$ in Fig. $2 \mathrm{~b}$, plotted from the pole to $40^{\circ}$, the latter corresponding to a radial distance of $\sim 2 R_{S}$ in the equatorial plane. The solid line shows values for the Northern Hemisphere, while the dot-dashed line shows values for the Southern Hemisphere (plotted versus co-latitude with respect to the southern pole). The difference is due to the north-south field asymmetry associated with the $g_{2}^{0}$ quadrupole term. Field line mapping between the equatorial plane and the northern 
and southern ionospheres then reduces to locating points of equal $F$ value on these surfaces.

In order to calculate the ionospheric currents we also need to know the magnitude and direction of the magnetic field in the ionosphere. We thus note that the components of the internal field consistent with Eq. (1) are

$$
\begin{aligned}
& B_{\text {int }_{r}}(r, \theta)=2 g_{1}^{0} \cos \theta\left(\frac{R_{S}}{r}\right)^{3}+\frac{3}{2} g_{2}^{0}\left(3 \cos ^{2} \theta-1\right)\left(\frac{R_{S}}{r}\right)^{4} \\
& +2 g_{3}^{0} \cos \theta\left(5 \cos ^{2} \theta-3\right)\left(\frac{R_{S}}{r}\right)^{5}
\end{aligned}
$$

and

$$
\begin{aligned}
& B_{\text {int }_{\theta}}(r, \theta)=g_{1}^{0} \sin \theta\left(\frac{R_{S}}{r}\right)^{3}+3 g_{2}^{0} \sin \theta \cos \theta\left(\frac{R_{S}}{r}\right)^{4} \\
& +\frac{3}{2} g_{3}^{0} \sin \theta\left(5 \cos ^{2} \theta-1\right)\left(\frac{R_{S}}{r}\right)^{5},
\end{aligned}
$$

from which the ionospheric magnetic field, $\boldsymbol{B}_{i}(\theta)=$ $B_{i_{r}}(\theta) \hat{\boldsymbol{r}}+B_{i_{\theta}}(\theta) \hat{\theta}$, can be found by substituting $r=R_{i}(\theta)$ from Eq. (3).

\subsection{Ionospheric Pedersen current}

Analysis of the Voyager spacecraft plasma velocity data yields models for the angular velocity of magnetospheric flux shells, $\omega=\omega(F)$, which determine the angular velocity as a function of co-latitude in the ionosphere through the mapping procedure discussed above. The specific angular velocity models we employ will be derived below in Sect. 3. Here, following prior derivations in the jovian context given, for example, by Hill (1979, 2001), Vasyliunas (1983), and Cowley and Bunce (2001), we first derive theoretical expressions for the horizontal ionospheric and fieldaligned currents which are applicable to the general case. In the derivation we distinguish three angular velocities, illustrated in Fig. 1. These are the angular velocity of the plasma on a given flux shell, $\omega$, as above, the angular velocity of the planet, $\Omega_{S}$ (equal to $1.638 \times 10^{-4} \mathrm{rad} \mathrm{s}^{-1}$ ), and the angular velocity of the neutral atmosphere in the Pedersen conducting layer of the ionosphere, $\Omega_{S}^{*}$. The value of $\Omega_{S}^{*}$ may differ from $\Omega_{S}$ due to the frictional torque associated with ionneutral collisions (e.g. Huang and Hill (1989) in the jovian context). We may thus expect $\Omega_{S}^{*}$ to lie at values intermediate between $\omega$ and $\Omega_{S}$, so we can write for some $0<k<1$ (whose value is at present uncertain)

$\left(\Omega_{S}-\Omega_{S}^{*}\right)=k\left(\Omega_{S}-\omega\right)$.

The angular velocity of the plasma in the neutral atmosphere rest frame is then

$\left(\Omega_{s}^{*}-\omega\right)=(1-k)\left(\Omega_{S}-\omega\right)$,

where positive values for sub-corotation of the plasma $(\omega<$ $\Omega_{s}$ ) indicate westward motion of the plasma in the neutral atmosphere rest frame. The corresponding ionospheric electric field in this frame is meridional, directed equatorward in both hemispheres, perpendicular to the ionospheric magnetic field (which is wholly poloidal according to the above model), and given by

$$
E_{i}(\theta)=\rho_{i}\left(\Omega_{S}^{*}-\omega\right) B_{i}=(1-k) R_{i}(\theta) \sin \theta\left(\Omega_{S}-\omega\right) B_{i}
$$

In Eq. (6) use has been made of Eq. (5b), $\rho_{i}=R_{i}(\theta) \sin \theta$ is the perpendicular distance from the spin (magnetic) axis, $R_{i}(\theta)$ is given by Eq. (3), and $B_{i}$ is the magnetic field strength in the ionosphere evaluated from Eq. (4) at radial distance $r=R_{i}(\theta)$ (i.e. $B_{i}=\left|\boldsymbol{B}_{i}\right|=\sqrt{B_{i_{r}}^{2}+B_{i_{\theta}}^{2}}$ ). The height-integrated Pedersen current intensity $i_{P}$ is then obtained by multiplying Eq. (6) by the height-integrated Pedersen conductivity $\Sigma_{P}$ to obtain

$$
\begin{aligned}
i_{P}(\theta) & =\Sigma_{P} E_{i}(\theta) \\
= & \Sigma_{P}^{*} \Omega_{S} B_{i p} R_{i}(\theta) \sin \theta\left(1-\frac{\omega}{\Omega_{S}}\right)\left(\frac{B_{i}}{B_{i p}}\right) .
\end{aligned}
$$

In Eq. (7) we have introduced the 'effective' value of the height-integrated ionospheric Pedersen conductivity (e.g. Huang and Hill, 1989)

$$
\Sigma_{P}^{*}=(1-k) \Sigma_{P},
$$

reduced from the true value by 'slippage' of the neutral atmosphere, where $k$ is defined by Eq. (5a). We have also introduced $B_{i p}$, the ionospheric field strength of the dipole term alone at the poles, given by

$$
B_{i p}=2 g_{1}^{0}\left(\frac{R_{S}}{R_{p}}\right)^{3} \approx 54760 \mathrm{nT},
$$

which is used as a convenient scale value of the polar ionospheric field strength. The last (magnetic field) term on the right-hand side of Eq. (7) is thus a factor of order unity describing the variation of the ionospheric field strength due to the presence of non-dipole terms and to changes in latitude.

A further order unity correction factor needs to be introduced, however, due to the fact that the magnetic field is not in general 'vertical' with respect to the ionospheric surface defined by Eq. (3), such that the Pedersen current, which is orthogonal to the magnetic field, does not flow exactly 'horizontally' within the conducting layer. We assume that this causes a small polarisation of the conducting layer, creating a small current in the direction of the magnetic field which is such as to make the total ionospheric current exactly 'horizontal' i.e. flowing wholly along the conducting layer. The horizontal Pedersen current intensity, as we will term it, is then given by

$i_{h P}(\theta)=i_{P}\left(\frac{B_{i}}{\left|B_{i_{n}}\right|}\right)$,

where $\left|B_{i_{n}}\right|$ is the modulus of the ionospheric field component which is normal to the conducting layer. The outward unit normal at co-latitude $\theta$ to the surface defined by Eq. (3) is

$\hat{\boldsymbol{n}}=n_{r}, \hat{\boldsymbol{r}}+n_{\theta} \hat{\boldsymbol{\theta}}=\frac{\left[\left(1+\varepsilon \cos ^{2} \theta\right) \hat{\boldsymbol{r}}-(\varepsilon \sin \theta \cos \theta) \hat{\boldsymbol{\theta}}\right]}{\left[\left(1+\varepsilon \cos ^{2} \theta\right)^{2}+(\varepsilon \sin \theta \cos \theta)^{2}\right]^{1 / 2}}$, 


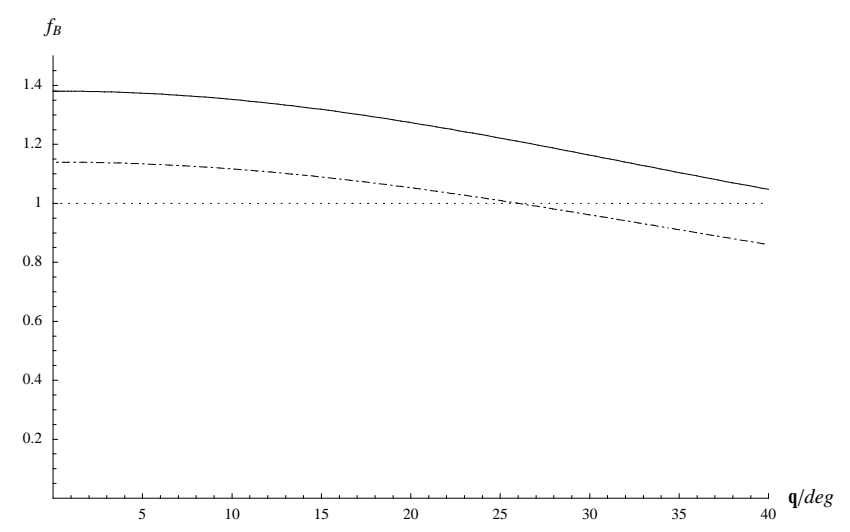

Fig. 3. Plot of the magnetic field factor $f_{B}$ appearing in the expression for the horizontal ionospheric current (Eq. 13), given by Eq. (14). This term contains the effects on the current due to nonvertical fields, non-dipole fields, and the field variation with magnetic latitude. The solid line shows values for the Northern Hemisphere, while the dot-dashed line shows values for the Southern Hemisphere, plotted versus co-latitude $\theta$ from the respective poles. The dotted line shows unity for purposes of comparison.

so that

$B_{i_{n}}=B_{i_{r}} n_{r}+B_{i_{\theta}} n_{\theta}$

where, as indicated above, $B_{i_{r}}$ and $B_{i_{\theta}}$ are the field components in the ionosphere obtained by putting $r=R_{i}(\theta)$ in Eq. (4). We thus finally obtain

$i_{h P}(\theta)=\Sigma_{P}^{*} \Omega_{S} B_{i p} R_{i}(\theta) \sin \theta\left(1-\frac{\omega}{\Omega_{S}}\right) f_{B}(\theta)$,

where

$f_{B}(\theta)=\left(\frac{B_{i}^{2}}{B_{i p}\left|B_{i_{n}}\right|}\right)$

is a factor of order unity which contains the effects of the non-vertical field, the non-dipole terms, and the variation of the field with latitude. This factor reduces to that given by Vasyliunas (1983) (see his Eq. 11.122) for the case of a spherical planet and dipole field alone. The value of $f_{B}$ is plotted versus $\theta$ in Fig. 3, where the solid line shows values for the Northern Hemisphere, while the dot-dashed line shows values for the Southern Hemisphere (plotted versus co-latitude with respect to the southern pole). Unity is shown by the dotted line. It can be seen that the term does not change the magnitude of the current very significantly in the Southern Hemisphere, while in the Northern Hemisphere it increases the value typically by a few tens of percent.

\subsection{Field-aligned currents}

We now wish to calculate the ionospheric field-aligned current density just above the current layer, $j_{\| i}(\theta)$, obtained from the divergence of the horizontal current given by
Eq. (13). We first define the azimuth-integrated horizontal Pedersen current $I_{h P}(\theta)$ as

$I_{h P}(\theta)=2 \pi \rho_{i} i_{h P}(\theta)=2 \pi R_{i}(\theta) \sin \theta i_{h P}(\theta)$.

Current continuity then requires that

$I_{h P}(\theta+d \theta)-I_{h P}(\theta)=-2 \pi R_{i}(\theta) \sin \theta d s j_{i_{n}}$,

where $d s$ is the length of arc in the ionosphere between $\theta$ and $\theta+d \theta$, given by

$d s=\frac{R_{i}(\theta) d \theta}{n_{r}}$,

where $n_{r}$ is the radial component of the outward unit normal to the ionosphere given by Eq. (11), and $j_{i_{n}}$ is the current density normal to the ionosphere, positive outwards, related to the ionospheric field-aligned current density by

$j_{i_{n}}=\frac{B_{i_{n}}}{B_{i}} j_{\| i}$

where $B_{i_{n}}$ is the field component normal to the ionospheric layer given by Eq. (12). Substituting Eqs. (17) and (18) into Eq. (16) and taking the limit then yields

$j_{\| i}(\theta)=-\frac{n_{r}}{2 \pi R_{i}^{2}(\theta) \sin \theta}\left(\frac{B_{i}}{B_{i_{n}}}\right) \frac{d I_{h P}}{d \theta}$.

We note in passing that the ionospheric Hall current flows eastward, carried by ions which are collisionally-coupled to the neutral atmosphere, and is axisymmetric within the approximations employed here. These currents do not, therefore, contribute to the divergence of the horizontal ionospheric current, nor hence to the field-aligned current density.

The nature of the overall current system envisaged is shown by the dashed lines in Fig. 1, appropriate to the case of sub-corotation of the magnetospheric plasma. In this case the ionospheric current flows equatorward in both hemispheres as indicated above, and closes via currents which flow radially outward in the equatorial magnetosphere. The $\boldsymbol{j} \times \boldsymbol{B}$ related torque associated with the ionospheric Pedersen current balances the torque imposed by ion-neutral collisions in the Pedersen layer. The equal and opposite $\boldsymbol{j} \times \boldsymbol{B}$-related torque associated with the magnetospheric current supplies angular momentum to the equatorial plasma that is either newly created in situ from neutral gas, or that is diffusing outward from internal sources (e.g. Richardson, 1992; Richardson et al., 1998). Overall, the current system is associated with the transfer of planetary angular momentum from the atmosphere to the equatorial magnetospheric plasma. The field-aligned currents which connect the field-perpendicular currents in the ionosphere and magnetosphere flow downward into the ionosphere at high latitudes, and upward out of the ionosphere at lower latitudes. It is thus the lower-latitude region of upward-directed field-aligned current that is potentially associated with accelerated auroral electron precipitation. 


\subsection{Auroral acceleration}

In order to assess whether the upward currents require downward field-aligned acceleration of magnetospheric electrons, we must compare their magnitude with the maximum current density that can be carried without field-aligned acceleration. This is given by

$j_{\| i 0}=e N\left(\frac{W_{t h}}{2 \pi m_{e}}\right)^{1 / 2}$,

corresponding to the condition of a full downward-going loss-cone in the magnetospheric electron population, and an empty upward-going loss-cone. In Eq. (20) $N$ is the number density of the magnetospheric electrons (assumed isotropic), $W_{t h}$ is their thermal energy, and $m_{e}$ and $e$ are the electron mass and charge. The corresponding precipitated electron energy flux is

$E_{f 0}=2 N W_{t h}\left(\frac{W_{t h}}{2 \pi m_{e}}\right)^{1 / 2}$.

If the required field-aligned current density is larger than that given by Eq. (20), then a field-aligned voltage must be present to accelerate magnetospheric electrons into the atmosphere. According to the kinetic theory presented by Knight (1973) (see also the discussion in Cowley and Bunce, 2001), the minimum field-aligned voltage required is

$\Phi_{\text {min }}=\frac{W_{t h}}{e}\left[\left(\frac{j_{\| i}}{j_{\| i 0}}\right)-1\right]$.

In addition, the minimum distance of the 'top' of the voltage drop, determined by the requirement that a large enough flux of magnetospheric electrons is present to form the current, is given by

$$
\left(\frac{r_{\min }}{R_{i}(\theta)}\right) \approx\left(\frac{j_{\| i}}{j_{\| i 0}}\right)^{1 / 3} .
$$

Here we have assumed as a sufficient approximation that the field strength falls as the inverse cube of the distance along the polar field lines. The corresponding enhanced precipitating electron energy flux is

$E_{f}=\frac{E_{f 0}}{2}\left[\left(\frac{j_{\| i}}{j_{\| i 0}}\right)^{2}+1\right]$,

following Lundin and Sandahl (1978).

Voyager plasma observations in the quasi-symmetric middle and inner regions of Saturn's magnetosphere show that the electron distribution consists of two populations, a cold dense population, and a warm tenuous population (Sittler et al., 1983). However, the cold population is electrostatically trapped by the cool ions in the vicinity of the equatorial plane, where they maintain charge neutrality, while the warm tenuous electrons extend approximately uniformly along the field lines to the ionosphere. It is thus the latter population which is germane to the issue of the aurora. Analyses presented by Richardson and Sittler (1990) and Richardson
(1995) indicate that typical parameters for this population are $N \approx 0.2 \mathrm{~cm}^{-3}$ and $W_{t h} \approx 150 \mathrm{eV}$. Substitution of these values into Eqs. (20) and (21) then yields $j_{\| i 0} \approx 66 \mathrm{nA} \mathrm{m}^{-2}$ and $E_{f 0} \approx 0.02 \mathrm{~mW} \mathrm{~m}^{-2}$. Assuming the latter energy flux is converted with $\sim 20 \%$ efficiency into $\sim 10 \mathrm{eV}$ UV photons, the corresponding auroral luminosity is $\sim 0.2 \mathrm{kR}$. This value is well below the sensitivity, for example, of the recent Hubble observations of Saturn's auroras (Trauger et al., 1998). In order to raise the luminosity towards the detectable range, say above $\sim 1 \mathrm{kR}$, requires current densities which exceed the value given by Eq. (20) by factors of at least $\sim 3$, that is we require field-aligned current densities $\sim 200 \mathrm{nA} \mathrm{m}^{-2}$ or higher. Model values based on the theory presented above are calculated in Sect. 4, following consideration in the next section of the physical parameters which have been determined from Voyager flyby data.

\section{Model parameters}

To determine the system currents based on Eqs. (13) and (19), we require, in addition to the magnetic model outlined above, a model of the angular velocity of the magnetospheric plasma, and a value for the effective height-integrated Pedersen conductivity of Saturn's ionosphere. These have been determined empirically from Voyager flyby data. In this section we show how the angular velocity profile has been derived from Voyager plasma data. We also summarise the results of a companion paper by Bunce et al. (2003), which discusses the value of the effective Pedersen conductivity as determined from an analysis of Voyager magnetic field data.

\subsection{Plasma angular velocity profiles}

We first consider the profiles of the plasma angular velocity, $\omega$, which have been determined from the Voyager-1 and -2 PLS instrument observations presented by Richardson (1986) and Richardson and Sittler (1990). These data show that, overall, the angular velocity of the plasma declines from near-rigid corotation in the inner magnetosphere, to values of around a half of rigid corotation in the outer regions considered here. Similar qualitative results were reported earlier by Frank et al. (1980) from analysis of Pioneer-11 plasma data, but it was not possible to derive a detailed angular velocity profile in that case. In addition to the large-scale variation, there are also indications of smaller scale features. During the inbound passage of the Voyager-1 spacecraft, in particular, localised reductions in the angular velocity were observed along the near-radial spacecraft trajectory on equatorial spatial scales of $\sim 1-2 R_{S}$, possibly associated with the orbits of the moons Dione and Rhea. Here, we have modelled and investigated the effects of both the large- and the small-scale variations, assuming that both are axially symmetric (or at least axially extended regarding the small-scale features) as a first approximation.

Briefly, the value of the flux function has been evaluated along the Voyager spacecraft trajectories using Eqs. (1) and 

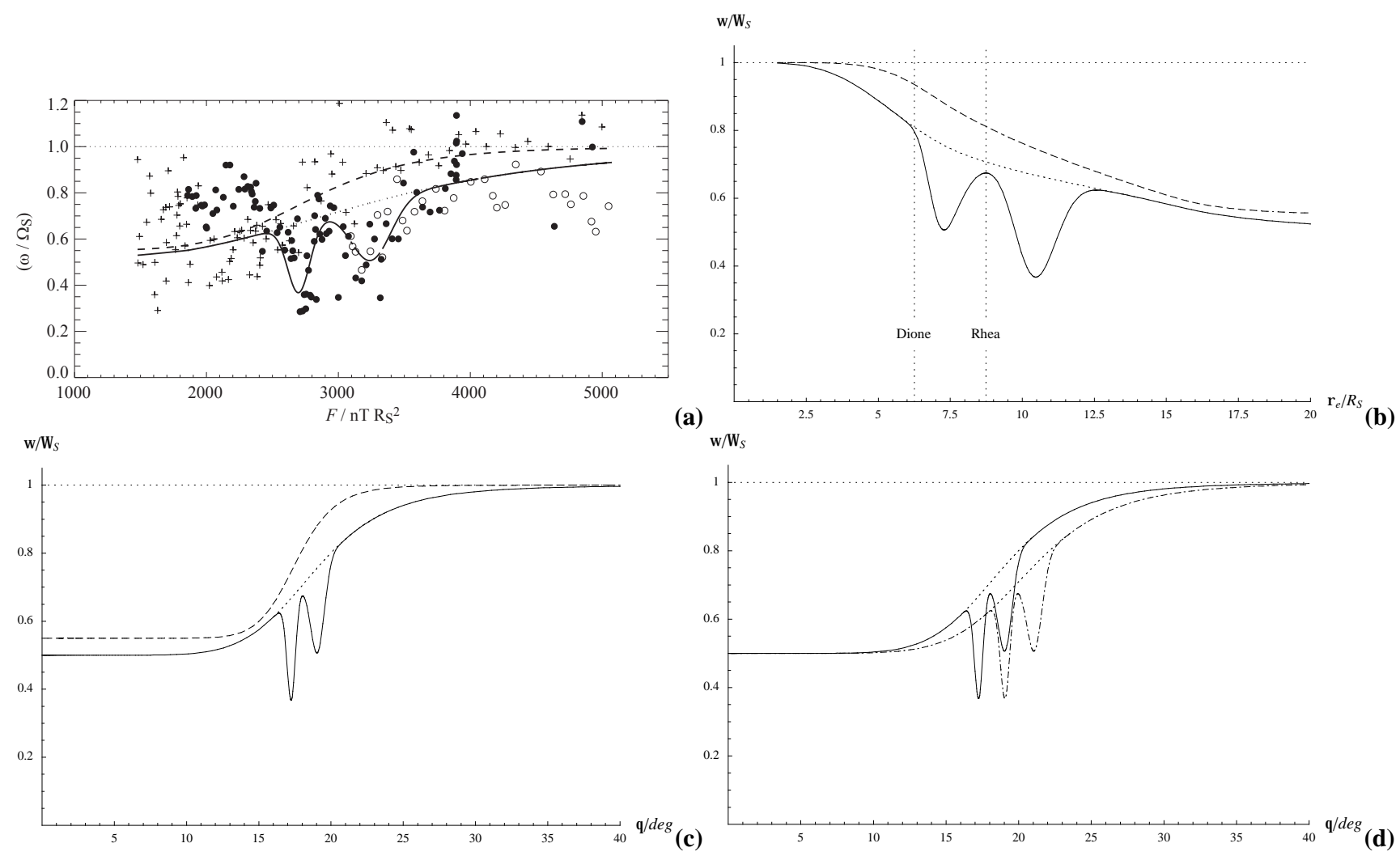

Fig. 4. (a) Plasma angular velocity values (normalised to Saturn's angular velocity $\Omega_{S}$ ) determined from Voyager PLS data, are shown plotted versus the magnetic field flux function $F$ evaluated along the spacecraft trajectories. The model magnetic field is given by the sum of the internal planetary field given by Eq. (1), and the Connerney et al. (1983) ring current model determined from fits to the Voyager magnetic field data, given by Eq. (2). Solid and open circles show data from the inbound and outbound passes of Voyagers-1 and -2 , respectively, while crosses show data from the inbound pass of Voyager-2. The curves show model fits to these data. The dashed curve shows a fit to the Voyager-2 data using Eq. (25) alone, with $\left(\omega / \Omega_{S}\right)_{\infty}=0.55, F_{O}=2800 \mathrm{nT} R_{S}^{2}$, and $n=7$. The solid curve shows a fit to the Voyager-1 profile using Eq. (25) with $\left(\omega / \Omega_{S}\right)_{\infty}=0.5, F_{O}=3200 \mathrm{nT} R_{S}^{2}$, and $n=4$, together with Eq. (26) with two terms given by $\left(\Delta \omega / \Omega_{S}\right)_{1}=0.25, F_{1}=3250 \mathrm{nT} R_{S}^{2}$, and $\Delta F_{1}=210 \mathrm{nT} R_{S}^{2}$, and $\left(\Delta \omega / \Omega_{S}\right)_{2}=0.3, F_{2}=2700 \mathrm{nT} R_{S}^{2}$, and $\Delta F_{2}=120 \mathrm{nT} R_{S}^{2}$. The dotted curve shows the Voyager-1 profile with the small-scale features omitted, given by Eq. (25) alone. The horizontal dotted line shows the condition for rigid corotation of the plasma, $\left(\omega / \Omega_{S}\right)=1$. (b) Plot of the model $\left(\omega / \Omega_{S}\right)$ profiles mapped along field lines into the equatorial plane and shown as a function of radial distance $\rho_{e}$ from the planet, normalised to the conventional Saturn radius $R_{S}$. The line format is the same as in (a). The radial distances of the orbits of the moons Dione and Rhea are indicated by the vertical dotted lines, showing their relationship to the 'dips' in the Voyager-1 profile. (c) Corresponding profiles in the Northern Hemisphere plotted versus co-latitude $\theta$, using the same line format as (a) and (b). (d) Comparison of Voyager-1 $\left(\omega / \Omega_{S}\right)$ profiles in the Northern (solid and dotted curves) and Southern (dot-dashed and dotted curves) Hemispheres.

(2) above, and the observed normalised plasma angular velocity $\left(\omega / \Omega_{S}\right)$ has been plotted versus $F$. The results are shown in Fig. 4a. The filled circles are data from the inbound pass of Voyager- 1 spanning the radial distance range $\sim 5-17 R_{S}$ at $\sim 13: 00-15: 00 \mathrm{LT}$, the open circles are partial data from the outbound pass of Voyager- 1 spanning $\sim 4-8 R_{S}$ at $\sim 02: 00 \mathrm{LT}$, while the crosses are data from the inbound pass of Voyager-2 spanning $\sim 4-20 R_{S}$ at $\sim 13: 00-15: 00 \mathrm{LT}$. Data from other portions of the trajectories are not available principally due to unfavourable instrument orientation with respect to the flow. If we consider the Voyager- 2 data first, it can be seen that the plasma is near-rigidly corotating (horizontal dotted line) at large values of $F$ corresponding to small radial distances from the planet in the equatorial plane, be- fore falling to values scattered around half of rigid corotation at smaller values of $F$, corresponding to large distances. The change occurs relatively abruptly near $F \approx 2800 \mathrm{nT} R_{S}^{2}$, corresponding to a distance of $\sim 10 R_{S}$ in the equatorial plane. To represent this behaviour we have fitted a function of the form

$$
\left(\frac{\omega}{\Omega_{S}}\right)=\left(\frac{\omega}{\Omega_{S}}\right)_{\infty}+\left(1-\left(\frac{\omega}{\Omega_{S}}\right)_{\infty}\right) \frac{1}{\left(1+\left(F_{o} / F\right)^{n}\right)},
$$

the fitting being done by eye, guided by a simultaneous evaluation of the RMS deviation per point between the data and the line. The function is such that the plasma rigidly corotates at small radial distances, i.e. $\left(\omega / \Omega_{S}\right) \rightarrow 1$ as $F \rightarrow \infty$, while the angular velocity monotonically decreases 
to $\left(\omega / \Omega_{S}\right)_{\infty}$ at large distances where $F \rightarrow 0$. Parameter $F_{o}$ determines the radial location where the angular velocity change occurs, while parameter $n$ determines the gradient of the change, becoming sharper with increasing $n$. The values of the parameters determined for Voyager- 2 are $\left(\omega / \Omega_{S}\right)_{\infty}=0.55, F_{o}=2800 \mathrm{nT} R_{S}^{2}\left(9.7 R_{S}\right.$ in the equatorial plane), and $n=7$, which is shown as the dashed line in Fig. 4a. The RMS deviation between the model profile and the Voyager-2 data is $\left(\delta \omega / \Omega_{S}\right) \sim 0.15$.

The Voyager-1 data overall are more scattered, but for simplicity we have represented their large-scale behaviour by a function of the same form. In this case we have considered the inbound and outbound data together, particularly given the paucity of inbound data at large values of $F$, and noting that axial symmetry should be a reasonable approximation in the inner regions of the magnetosphere. The combined data then indicate that near-rigid corotation breaks down at smaller radial distances (larger $F$ values) for Voyager-1 than for Voyager-2, as pointed out previously by Richardson (1986), and declines with decreasing $F$ more gradually. The values of the parameters chosen to represent this behaviour are $\left(\omega / \Omega_{S}\right)_{\infty}=0.5, F_{o}=3200 \mathrm{nT} R_{S}^{2}$, (corresponding to $7.4 R_{S}$ in the equatorial plane), and $n=4$, as shown by the dotted line in Fig. 4a (which is in some portions overlain by the solid line to be described below). This profile fits the data reasonably well for $F$ values above $\sim 2400 \mathrm{nT} R_{S}^{2}$ (radial distances less than $\sim 13 R_{S}$ in the equatorial plane), but does not properly represent the somewhat increased angular velocity values observed at smaller $F$ values. It also does not represent the small-scale features, mentioned above, in which the angular velocity is significantly reduced below that indicated by the dotted curve, which are observed in the vicinity of $\sim 3250$ and $\sim 2700 \mathrm{nT} R_{S}^{2}$. These $F$ values map to distances of $\sim 7$ and $\sim 10 R_{S}$ in the equatorial plane, and thus to locations just outside the orbits of the moons Dione and Rhea (as also pointed out previously by Richardson, 1986). These moons orbit in the equatorial plane at distances of 6.25 and $8.74 R_{S}$, respectively, but were not located close to the spacecraft at the time it crossed their respective paths. These features have been represented by adding small-scale Gaussian terms to Eq. (25) of the form

$$
\left(\frac{\Delta \omega}{\Omega_{S}}\right)=-\sum_{n}\left(\frac{\Delta \omega}{\Omega_{S}}\right)_{n} \exp \left(-\left(\frac{F-F_{n}}{\Delta F_{n}}\right)^{2}\right),
$$

thus effectively assuming that the while the flow signatures are restricted in radial scale, as observed, they are nevertheless elongated in azimuth, as seems physically plausible in the corotation-dominated middle-magnetosphere region. The solid line shown in Fig. 4a represents the effect of adding two such terms to Eq. (25) (using the Voyager-1 parameters given above), with $\left(\Delta \omega / \Omega_{S}\right)_{1}=0.25, F_{1}=3250 \mathrm{nT} R_{S}^{2}$ (7.2 $R_{S}$ in the equatorial plane), and representing the dip outside Dione's orbit, and $\left(\Delta \omega / \Omega_{S}\right)_{2}=0.3, F_{2}=2700 \mathrm{nT} R_{S}^{2}$, (10.4 $R_{S}$ in the equatorial plane), and $\Delta F_{2}=120 \mathrm{nT} R_{S}^{2}$ representing the dip outside Rhea's orbit. There is little evidence for corresponding dips in the Voyager-2 data, and none have therefore been incorporated into the Voyager-2 model profile, which thus describes the large-scale variation alone.

In Fig. 4b we thus show the model angular velocity profiles mapped along model magnetic field lines to the equatorial plane. The solid line again shows the overall profile for Voyager-1, obtained by adding the small-scale dips given by Eq. (26) to the large-scale behaviour given by Eq. (25). As indicated above, the dips occur outside the orbits of the moons Dione and Rhea, marked by the vertical dotted lines, and have equatorial spatial scales of $\sim 1-2 R_{S}$. The dotted curve shows the large-scale Voyager-1 profile with the dips omitted. The dashed line similarly shows the profile for Voyager-2, given by Eq. (25) alone with modified parameters given above. In this case near-rigid corotation is maintained to larger distances, as noted above. In Fig. $4 \mathrm{c}$ we then show these profiles mapped along the field into the ionosphere in the Northern Hemisphere. The line format is the same as for Figs. $4 \mathrm{a}$ and $\mathrm{b}$. It can be seen that the main variations for Voyager- 1 occur in the co-latitude range between $\sim 15^{\circ}$ and $\sim 25^{\circ}$. The 'Rhea' and 'Dione' angular velocity dips are $\sim 1^{\circ}$ wide in the ionosphere, and map to $\sim 17^{\circ}$ and $\sim 19^{\circ}$, respectively. For Voyager-2, the main variations occur between $\sim 15^{\circ}$ and $\sim 20^{\circ}$. Figure $4 \mathrm{~d}$ compares the profiles in the Northern and Southern Hemispheres, where for clarity we show the Voyager-1 profile only. The Northern Hemisphere profile is shown by the solid (and dotted) line, while the Southern Hemisphere profile is shown by the dot-dashed (and dotted) line. These profiles are essentially similar, but the Southern Hemisphere profile is shifted equatorward by $\sim 2^{\circ}$ (in the relevant region) with respect to that in the north. In particular, the 'Rhea' and 'Dione' dips now map to $\sim 19^{\circ}$ and $\sim 21^{\circ}$, respectively. As in Fig. $2 b$, the relative shift is due to the effect of the quadrupole term of the internal field.

We should emphasise, however, that the angular velocity profiles shown in Figs. $4 \mathrm{c}$ and $\mathrm{d}$ are valid, supported by the Voyager observations, only for sufficiently large co-latitudes mapping to the quasi-axisymmetric middle and inner regions of the magnetosphere. If the equatorial profiles are taken to be valid to a radial distance of $15 R_{S}$, then the ionospheric profiles will correspondingly be valid for co-latitudes greater than $15.3^{\circ}$ in the north, and $16.8^{\circ}$ in the south. If, on the other hand, we extend the model range to $20 R_{S}$ in the equatorial plane, as shown in Figs. $2 \mathrm{a}$ and $4 \mathrm{~b}$, then this maps in the ionosphere to co-latitudes greater than $12.8^{\circ}$ in the north and $14.1^{\circ}$ in the south. Field lines at smaller co-latitudes then map to the vicinity of the magnetopause and into Saturn's magnetic tail where the solar wind interaction will become important, such that the model derived here ceases to be applicable.

\subsection{Effective ionospheric Pedersen conductivity}

The final parameter required for our model is the effective height-integrated Pedersen conductivity of Saturn's ionosphere, possibly reduced from the true value by atmospheric 'slippage', as discussed above. A number of estimates have been made of the true value based on observed and modelled 

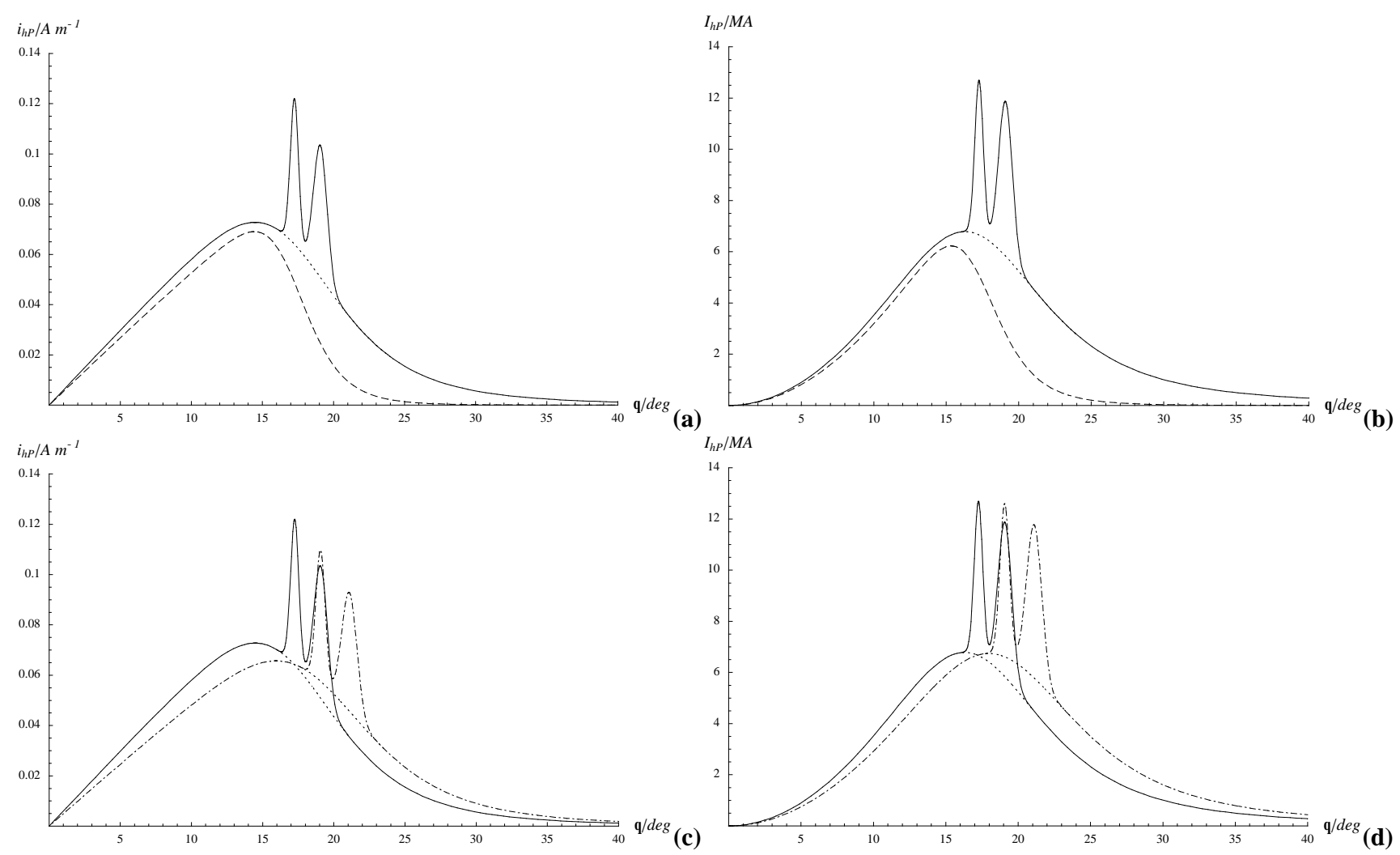

Fig. 5. (a) Plot of the equatorward-directed horizontal height-integrated ionospheric current intensity in the Northern Hemisphere versus co-latitude $\theta$, obtained from Eq. (13) with $\Sigma_{P}^{*}=1$ mho. The format follows Fig. 4, with the solid and dotted lines showing results using the Voyager-1 angular velocity models with and without small-scale features, and the dashed line showing results using the Voyager- 2 angular velocity model. (b) Corresponding plot of the azimuth-integrated horizontal ionospheric current in the Northern Hemisphere, obtained from Eq. (15). (c) Comparison of the current intensity curves in the Northern and Southern Hemispheres using the Voyager-1 angular velocity models. The solid line corresponds to the Northern Hemisphere, as shown in (a), while the dot-dashed line corresponds to the Southern Hemisphere. (d) Comparison of the azimuth-integrated current curves in the Northern and Southern Hemispheres using the Voyager-1 angular velocity models, in the same format as (c).

ionospheric electron density profiles, with values varying between $\sim 0.1$ and $\sim 100$ mho (Atreya et al., 1984; Connerney et al., 1983; Cheng and Waite, 1988). The appropriate value of $\Sigma_{P}^{*}$ may thus be considered to be very uncertain at the present time. However, an empirical estimate can be made using flyby magnetic field data. It can be seen from Fig. 1 that the current system considered here leads to the presence of azimuthal magnetic fields in the region above the ionosphere, which reverse in sense across the current-carrying region in the equatorial magnetosphere. Transfer of angular momentum from the atmosphere to the sub-corotating equatorial plasma thus leads to the field lines being bent back into a 'lagging' configuration, as mentioned above. Simple application of Ampère's law shows that the magnitude of the azimuthal field outside the equatorial current region is proportional to the azimuth-integrated horizontal Pedersen current, given by Eq. (15), which is flowing at the feet of the field lines in the corresponding hemisphere. For given models of the magnetic field and plasma angular velocity, this field is then proportional to the effective ionospheric Peder- sen conductivity. Comparison between measured and modelled azimuthal fields thus provides information on the effective conductivity.

This comparison has been carried out in a companion paper by Bunce et al. (2003), using the theory presented above applied to Voyager-1 and -2 magnetic field data. The results show a reasonable agreement between the measured and modelled fields, particularly in the inner region, for values $\Sigma_{P}^{*} \approx 1-2$ mho. However, other dynamical effects may also contribute to the measured azimuthal fields in this region (e.g. Connerney et al., 1983; Espinosa and Dougherty, 2000), such that it is not certain that all the observed fields (where it is in approximate accord with the theory) can be attributed to the magnetosphere-ionosphere coupling current system discussed here. In this case, the effective Pedersen conductivity must be equal to or less than the above values. The robust result of this study, therefore, is that the effective height-integrated Pedersen conductivity in the regions investigated here, mapping to the middle and inner magnetosphere, is of the order of $\sim 1$ mho or less. Values above 

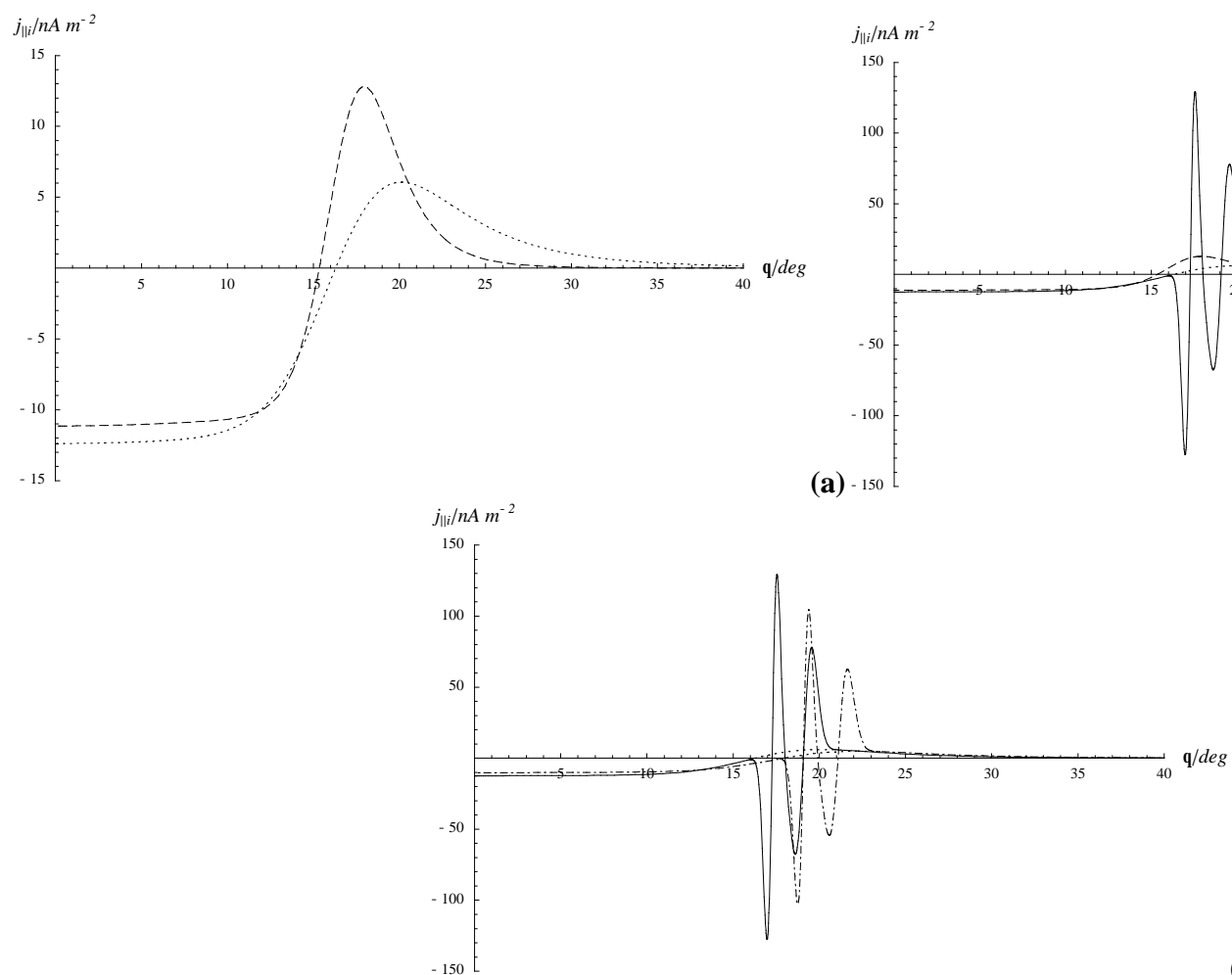

(b)

Fig. 6. (a) Plot of the ionospheric field-aligned current density in the Northern Hemisphere versus co-latitude $\theta$, obtained from Eq. (19) with $\Sigma_{P}^{*}=1$ mho. Only the currents associated with the large-scale variations in the angular velocity modelled by Eq. (25) are shown, such that the 'dips' in the Voyager-1 profile are ignored. The line format is the same as for Figs. 4 and 5, such that the profile for Voyager-1 (without 'dips') is shown dotted, while the Voyager-2 profile is shown dashed. (b) Shows the same Northern Hemisphere field-aligned current curves as in (a), but now also including the 'dips' in the Voyager-1 profile (solid line). The current scale has also been expanded in order to show the peaks associated with the small-scale Voyager-1 features. (c) Shows a comparison of the Voyager-1 field-aligned current profiles in the Northern and Southern Hemispheres, in the same format as Figs. 4 and 5.

$\sim 2$ mho, and of the order of $\sim 10-100$ mho, can certainly be ruled out. In this paper we have therefore taken the effective ionospheric Pedersen conductivity to be equal to 1 mho, such that the model currents (but not the auroral parameters derived from them) can be simply scaled to other values of the conductivity. We note, however, that we consider this value for $\Sigma_{P}^{*}$ to be at the top end of the range of likely values, such that the currents derived below are also regarded as being close to upper limits.

\section{Model results}

\subsection{Ionospheric Pedersen currents}

In this section we will now present results for the ionospheric and field-aligned currents determined from Eqs. (13), (15), and (19), using the magnetic field model outlined in Sect. 2, the Voyager angular velocity profiles discussed in Sect. 3, and $\Sigma_{P}^{*}=1$ mho. Figure 5a shows the horizontal ionospheric Pedersen current intensity $i_{h P}$ obtained from Eq. (13), plotted versus co-latitude $\theta$ in the Northern Hemisphere. As in Fig. 4, the solid and dotted lines correspond to the Voyager-1 angular velocity profiles with and without small-scale dips, while the dashed line corresponds to the Voyager-2 profile. Initially ignoring the effects due to the small-scale features in the Voyager-1 profile, it can be seen that in each case the overall current profile increases near-linearly with co-latitude near the pole, before peaking at $\sim 0.7 \mathrm{~A} \mathrm{~m}^{-1}$ near $\sim 15^{\circ}$, and then falling over a colatitude range of $\sim 5^{\circ}$ (for the Voyager- 2 model) to $\sim 10^{\circ}$ (for Voyager-1). The near-linear rise in the current intensity at small co-latitudes occurs in the (generally inapplicable) region where the model angular velocities are nearly constant (see Fig. 4c). This is due to the consequent near-linear increase in this region of the ionospheric field line velocity (and electric field) in the neutral atmosphere rest frame with increasing distance from the pole. The subsequent peaking and decreasing of the current is due to the increase in the plasma angular velocity with increasing co-latitude, and the approach to near-rigid corotation. The latter takes place at smaller co-latitudes for Voyager-2 than for Voyager-1, corresponding to larger distances in the equatorial plane, as is evident in Figs. $4 \mathrm{~b}$ and c. It can also be seen, however, that the inclusion of small-scale features in the Voyager-1 angu- 
lar velocity profile has a dramatic effect on the corresponding current profile, resulting in narrow peaks in the current at the centres of the velocity dips lying outside the mapped orbits of Rhea and Dione. The overall current maxima at these features are $\sim 1.2$ and $\sim 1.0 \mathrm{~A} \mathrm{~m}^{-1}$, respectively.

Figure $5 \mathrm{~b}$ similarly shows the azimuth-integrated horizontal Pedersen current in the Northern Hemisphere, given by Eq. (15). Again, initially ignoring the effect of the smallscale features in the Voyager-1 profile, it can be seen that the currents initially grow with increasing co-latitude in the (generally inapplicable) region where the model plasma angular velocities are nearly constant, essentially as the square of the distance from the pole, before peaking at $\sim 6 \mathrm{MA}$ near $\sim 15^{\circ}$ co-latitude, and then falling with increasing co-latitude as before. The small-scale features in the Voyager-1 angular velocity model again result in substantial effects, however, producing individual azimuth-integrated current peaks of $\sim 13$ and $\sim 12 \mathrm{MA}$, respectively, outside the mapped orbits of Rhea and Dione.

Figures $5 \mathrm{c}$ and $\mathrm{d}$ show comparisons of the Northern and Southern Hemisphere profiles of the Voyager-1 horizontal Pedersen current intensity and azimuth-integrated horizontal Pedersen current, respectively, in the same format as Fig. 4d. It can be seen in Fig. 5c that the Southern Hemisphere current intensity profile is of modestly lower amplitude than that in the north, and, as before, is displaced equatorward by $\sim 2^{\circ}$ in the region of central interest, between $\sim 15^{\circ}$ and $\sim 30^{\circ}$. It may be noted that this displacement has the effect of placing the 'Dione' peak in the Northern Hemisphere at nearly the same co-latitude as the 'Rhea' peak in the south. While the peak current intensities are thus less in the south than in the north, this is offset in the azimuth-integrated current curves shown in Fig. $5 \mathrm{~d}$ by the equatorward displacement of the peaks to larger radial distances from the axis. This effect increases the azimuth-integrated current, such that the peak values of corresponding features are very nearly the same in the south as in the north.

\subsection{Field-aligned currents}

The field-aligned current above the ionosphere, given by Eq. (19), relates specifically to the latitude gradient of the azimuth-integrated horizontal Pedersen current shown in Figs. $5 \mathrm{~b}$ and d, and is shown in Fig. 6 in a corresponding format. Figure $6 a$ shows the field-aligned current in the Northern Hemisphere associated with the large-scale angular velocity variations modelled by Eq. (25), thus ignoring the 'dips' in the Voyager-1 angular velocity profile. Only the 'dotted' profile for Voyager-1 is thus shown, together with the profile for Voyager-2, shown as a dashed profile. It can be seen that these currents are negative, i.e. directed downward into the ionosphere, in the (generally inapplicable) region near the pole where the azimuth-integrated horizontal Pedersen currents are increasing with increasing latitude. The magnitude is $\sim 10 \mathrm{nA} \mathrm{m}^{-2}$. The currents then reverse sense to positive, i.e. directed upward out of the ionosphere, near $\sim 15^{\circ}$ where the azimuth-integrated current maximizes, be- fore peaking and falling with increasing co-latitude. The peak upward field-aligned current density for the Voyager1 model (without small-scale features) is $\sim 6 \mathrm{nA} \mathrm{m}^{-2}$ located at $\sim 20^{\circ}$ co-latitude, while that for the Voyager- 2 model is $\sim 13 \mathrm{nA} \mathrm{m}^{-2}$ located at $\sim 18^{\circ}$ co-latitude. Current densities of such magnitude follow as a simple consequence of peak equatorward-directed azimuth-integrated currents of $\sim 6 \mathrm{MA}$ flowing out of the ionosphere in an equatorward latitude strip which is $\sim 5^{\circ}$ wide for the Voyager- 2 model and $\sim 10^{\circ}$ wide for Voyager-1. Comparison with the limiting current densities estimated in Sect. 2.3 then shows that these currents are well below those requiring field-aligned acceleration of warm magnetospheric electrons, by factors of $\sim 5-10$. No enhancement of the precipitating energy flux above the expected $\sim 0.2 \mathrm{kR}$ background of 'diffuse' emission is therefore expected on their account.

Figure $6 \mathrm{~b}$ shows the same curves as in Fig. 6a, but now also including the 'dips' in the Voyager-1 profile (solid line), and using a larger current density scale which is sufficient to display the additional features. Each of the angular velocity dips are associated with bipolar field-aligned current features in which the current flows downward at higher latitudes, and upward at adjacent lower latitudes. The peak current densities are $\sim 130 \mathrm{nA} \mathrm{m}^{-2}$ for the 'Rhea dip', and $\sim 70 \mathrm{nA} \mathrm{m}^{-2}$ for the 'Dione dip'. The difference between the two values results simply from the fact that the 'Rhea dip' has been modelled as marginally deeper and narrower than the 'Dione dip', based on the Voyager-1 data. Fieldaligned current densities of this order are again the simple consequence of additional azimuth-integrated horizontal ionospheric currents of $~ 6 \mathrm{MA}$ in each case growing and decaying in a latitude strip which is $\sim 1^{\circ}$ wide for the 'Rhea' feature, and $\sim 2^{\circ}$ wide for the 'Dione' feature (see Fig. $5 \mathrm{~b}$ ). It can then be seen that such currents do indeed require fieldaligned acceleration of magnetospheric electrons, since both exceed the $\sim 65 \mathrm{nA} \mathrm{m}^{-2}$ limit estimated in Sect. 2.3, though only marginally so for the 'Dione' feature. Before examining this aspect, however, we first show in Fig. 6c a comparison of the Voyager-1 field-aligned current profiles in the Northern and Southern Hemispheres, in the same format as Figs. $4 \mathrm{c}$ and $5 \mathrm{c}-\mathrm{d}$. This shows that the peak current densities are marginally less in the Southern Hemisphere than in the north, as a consequence of their equatorward displacement and marginally increased ionospheric area out of which essentially the same total current flows. The peak current densities in the Southern Hemisphere are $\sim 100 \mathrm{nA} \mathrm{m}^{-2}$ for the 'Rhea' feature, and $\sim 60 \mathrm{nA} \mathrm{m}^{-2}$ for the 'Dione' feature (which is thus now below that required for field-aligned acceleration).

\subsection{Auroral parameters}

The auroral acceleration parameters required by the fieldaligned currents associated with the small-scale angular velocity features in the Voyager-1 profile are shown on an expanded co-latitude scale for the Northern (solid lines) and Southern (dot-dashed line) Hemispheres in Fig. 7. Figure 7a 


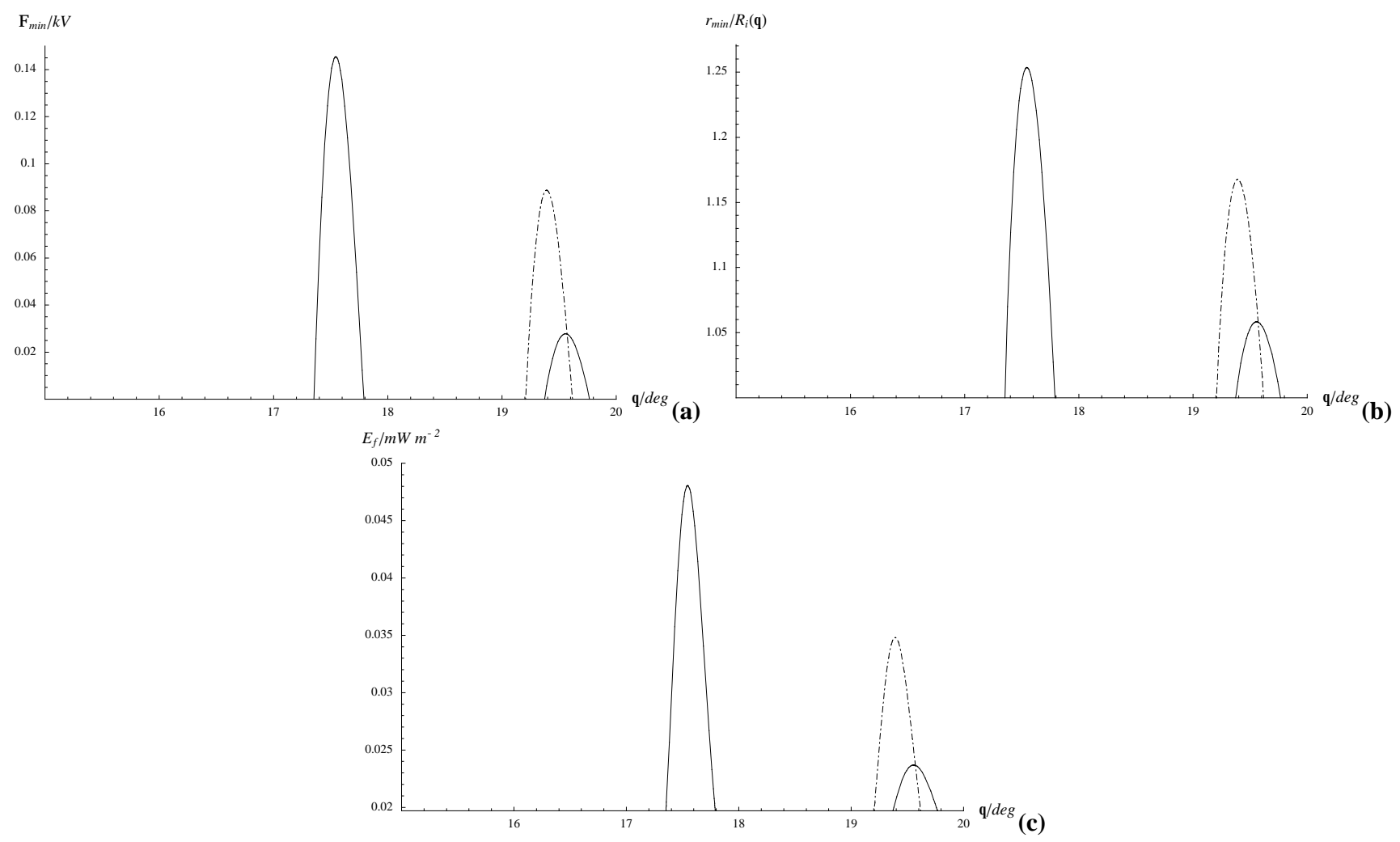

Fig. 7. Plots showing auroral electron acceleration parameters on an expanded co-latitude scale for the Voyager-1 angular velocity model (including small-scale features) and $\Sigma_{P}^{*}=1 \mathrm{mho}$, for the Northern Hemisphere (solid lines) and Southern Hemisphere (dot-dashed lines). These have been derived using Eqs. (20)-(24), with $N=0.2 \mathrm{~cm}^{-3}$ and $W_{t h}=150 \mathrm{eV}$. (a) Shows the minimum accelerating voltage obtained from Eq. (22). The two peaks in the Northern Hemisphere at $\sim 17.6^{\circ}$ and $\sim 19.6^{\circ}$ correspond to the 'Rhea'- and 'Dione'-related angular velocity features, respectively. The single peak in the Southern Hemisphere at $\sim 19.4^{\circ}$ corresponds to the 'Rhea' feature. The 'Dione' feature does not have sufficient current density to require field-aligned acceleration in the Southern Hemisphere. (b) Shows the minimum radial distance of the top of the acceleration region in planetary radii, obtained from Eq. (23). (c) Shows the energy flux of the precipitating auroral electrons, obtained from Eq. (24). The energy flux scale starts at the estimated level of the unaccelerated background precipitation from the magnetosphere, i.e. at $\sim 0.02 \mathrm{~mW} \mathrm{~m}^{-2}$. The associated $\mathrm{UV}$ auroral emission can be estimated in $\mathrm{kR}$ by multiplying the precipitated energy flux by a factor of $\sim 10$.

shows the minimum accelerating voltage required, obtained from Eq. (22), with $W_{t h} \approx 150 \mathrm{eV}$ and $j_{\| i 0} \approx 66 \mathrm{nA} \mathrm{m}^{-2}$, as indicated in Sect. 2.3 above. For the 'Rhea' feature, accelerating voltages are required in a region $\sim 0.4^{\circ}$ wide (i.e. a strip $\sim 400 \mathrm{~km}$ wide in the ionosphere), centred at $\sim 17.6^{\circ}$ colatitude in the north, and $\sim 19.4^{\circ}$ in the south. The peak voltages are $\sim 150$ and $\sim 90 \mathrm{~V}$, respectively. The 'Dione' feature requires accelerating voltages only in the Northern Hemisphere, as indicated above, peaking at $\sim 30 \mathrm{~V}$ at $\sim 19.6^{\circ}$. The minimum radial distance of the top of the acceleration region, given as a fraction of the local radius of the ionosphere by Eq. (23), is similarly shown in Fig. 7b. It can be seen that the acceleration region does not need to extend to great heights above the ionosphere in order to supply the flux of electrons required to carry the current. In particular, the accelerating voltages required by the 'Rhea' feature need extend only to distances of $\sim 0.25$ and $\sim 0.17$ planetary radii above the conducting layer in the Northern and Southern Hemispheres, respectively (corresponding to heights of $\sim 14000$ and $\sim 9500 \mathrm{~km}$, respectively). Figure $7 \mathrm{c}$ finally shows the precipitating electron energy fluxes given by Eq. (24) plotted in the same format, where the energy flux scale starts at the level of the background diffuse precipitation, i.e. at $E_{f 0} \approx 0.02 \mathrm{~mW} \mathrm{~m}^{-2}$. We recall that this corresponds to an estimated diffuse UV auroral emission of $\sim 0.2 \mathrm{kR}$. The peak energy flux associated with the Northern Hemisphere 'Rhea' feature at $\sim 17.6^{\circ}$ is just over double this value, at $\sim 0.05 \mathrm{~mW} \mathrm{~m}^{-2}$, thus corresponding to $\sim 0.5 \mathrm{kR}$. In the Southern Hemisphere this is reduced to $\sim 0.035 \mathrm{~mW} \mathrm{~m}^{-2}$, corresponding to $\sim 0.35 \mathrm{kR}$. The 'Dione' feature in the Northern Hemisphere is barely above the background level. We thus conclude that enhanced auroral emissions may sometimes accompany such small-scale features in the angular velocity profiles, particularly in the Northern Hemisphere, but that they are likely to be relatively dim, and elevated by only modest factors above the background level.

It should be remembered, however, that the latter conclusions depend on the effective value of the ionospheric Peder- 
sen conductivity, assumed to be equal to 1 mho in the results presented here. While the values of the currents shown in Figs. 5 and 6 simply scale in proportion to the conductivity, the auroral parameters shown in Fig. 7 do not. For example, if we increase the value of the conductivity to $\Sigma_{P}^{*}=2 \mathrm{mho}$, then the peak minimum accelerating voltage for the 'Rhea' feature increases to $\sim 450 \mathrm{~V}$ in the Northern Hemisphere and to $\sim 300 \mathrm{~V}$ in the Southern Hemisphere, while the minimum height of the top of the acceleration regions increases to $\sim 0.58$ planetary radii above the conducting layer in the Northern Hemisphere, and to $\sim 0.47$ planetary radii above the layer in the Southern Hemisphere. The peak precipitating energy flux also increases, to $\sim 0.16 \mathrm{~mW} \mathrm{~m}^{-2}$ in the Northern Hemisphere, corresponding to a $\sim 1.6 \mathrm{kR}$ aurora, and to $\sim 0.11 \mathrm{~mW} \mathrm{~m}^{-2}$ in the Southern Hemisphere, corresponding to a $\sim 1.1 \mathrm{kR}$ aurora. On the other hand, no acceleration at any of these features is required if the conductivity is less than $\sim 0.5 \mathrm{mho}$, such that in this case no sensible enhancement will occur above the background precipitation level.

\section{Summary and discussion}

In this paper we have estimated the magnitude and distribution of the horizontal Pedersen currents which are driven in Saturn's ionosphere by sub-corotation of the magnetospheric plasma, the calculation taking into account the detailed structure of Saturn's magnetic field as it is presently known from flyby data, as well as the flattened nature of the figure of the planet. The field-aligned currents which flow between the ionosphere and magnetosphere have then been calculated from the divergence of the ionospheric current. Estimation of these currents relies on the knowledge of two physical parameters, the first being the angular velocity of the plasma, and how it varies with radial distance in the equatorial magnetosphere and with co-latitude in the ionosphere, the second being the effective height-integrated Pedersen conductivity of Saturn's ionosphere, possibly reduced from the true value by neutral atmosphere 'slippage'. These parameters have been investigated using data from the flybys of the two Voyager spacecraft (the conductivity in a companion paper by Bunce et al., 2003), and the results have been applied in the calculations presented here. The region of validity of our results extends to distances of $\sim 15-20 R_{S}$ in the equatorial plane (depending, for example, on the degree of extension of the magnetosphere), corresponding to ionospheric colatitudes equatorward of $\sim 15^{\circ}$. In this region the plasma angular velocity falls, overall, from near-rigid corotation with the planet in the inner region, to about half of the rigid corotation at the outer boundary. In addition, evidence also exists in the Voyager data for small-scale features in the angular velocity profiles, $\sim 1-2 R_{S}$ in radial extent in the equatorial plane, centred near $\sim 7$ and $\sim 10 R_{S}$, just outside the orbits of Dione and Rhea, respectively. Such features have also been included in the models and their effects investigated, assuming that they represent azimuthally-elongated structures. In order to calculate the currents associated with these flows, we employed an effective ionospheric Pedersen conductivity of 1 mho, which is of the order suggested by the results presented in the companion paper, the specific value being chosen so that the results for the currents (but not the auroral parameters) can be simply scaled to other choices.

We first consider the effects associated with the large-scale variation of the plasma angular velocity. We find using the above models and values that the equatorward-directed horizontal ionospheric current peaks near the boundary of the region of applicability of the model, near $\sim 15^{\circ}$ co-latitude, and then falls toward zero in the equatorward region over a latitude range of $\sim 5^{\circ}-10^{\circ}$, depending on the details of the plasma angular velocity profile. Peak ionospheric current intensities are $\sim 0.06 \mathrm{~A} \mathrm{~m}^{-1}$ near the poleward boundary, corresponding to peak azimuth-integrated currents flowing in the magnetosphere-ionosphere coupling circuit of $\sim 6 \mathrm{MA}$. This current then flows out of the ionosphere along the field lines in the equatorward region, as the plasma approaches rigid corotation with the planet and the horizontal current drops toward zero. The field-aligned current flow is thus directed outward, from the ionosphere to the magnetosphere, throughout most of the region of validity of the model, with the possible exception of the outermost layers. The magnitude of the field-aligned current just above the ionosphere peaks at values of the order of $\sim 10 \mathrm{nA} \mathrm{m}^{-2}$, the exact value depending on the details, with the peak being located at $\sim 18^{\circ}-20^{\circ}$ co-latitude in the Northern Hemisphere, and $\sim 20^{\circ}-22^{\circ}$ in the Southern Hemisphere (with respect to the southern pole), the difference being due to the north-south field asymmetry associated with the internal quadrupole field term.

As at Jupiter, such rings of upward-directed field-aligned current are potentially associated with downward-accelerated electron precipitation, and consequent discrete auroral emission. However, using Voyager's plasma electron observations, we estimate that the limiting current density above which field-aligned electron acceleration is required is several tens of $\mathrm{nAm}^{-2}$. The limiting current is thus a considerable factor larger than the largest field-aligned current densities estimated here on the basis of the large-scale angular velocity models. Enhanced auroral precipitation above an expected diffuse background of a few tenths of a $\mathrm{kR}$ in association with this current system is thus not anticipated. Unlike the corresponding case at Jupiter, therefore, the results obtained here do not provide a basis for understanding the 'main auroral oval' emissions at Saturn, whose main features were outlined in the Introduction. Not only are the large-scale upward currents of insufficient intensity to produce an aurora, they also flow at too low a latitude to explain the observed emission, peaking at $\sim 20^{\circ}$ co-latitude as opposed to $\sim 10^{\circ}-15^{\circ}$ observed for the aurora (see the discussion in the Introduction). Furthermore, the auroral distribution expected from the corotation-enforcement current system would be expected to be relatively steady with time, and independent of local time to a first approximation, whereas the observed auroras appear to be very variable in time and to have a pronounced local time asymmetry, with peak dayside 
emissions occurring in the pre-noon sector (Trauger et al., 1998). All these considerations therefore point to one conclusion, that the 'main oval emissions' at Saturn, unlike those at Jupiter, are not associated with the corotation-enforcement current system. Rather, we may speculate, with previous authors, that these auroras are instead associated with solar wind-magnetosphere coupling currents, with the pre-noon enhancement then being related to the upward-directed 'region 1' currents in the dawn sector.

It is of interest to briefly consider the factors that produce such different results for Jupiter and Saturn. We first note that (i) the planetary angular velocities are comparable, as are the relative breakdowns of the plasma angular velocity from rigid corotation which are envisaged, and (ii) the planetary radii are also comparable, as are the co-latitudes at which the breakdown occurs. Taken together, this means that the ionospheric plasma velocities relative to the neutral atmosphere are expected to be roughly the same in the two systems. However, the ionospheric magnetic field strength is an order of magnitude larger at Jupiter than at Saturn, thus resulting in an electric field in the neutral atmosphere rest frame which is also larger by an order of magnitude. This, combined with an effective ionospheric Pedersen conductivity, which is taken as roughly comparable at the two planets, results in ionospheric Pedersen currents which are an order of magnitude larger at Jupiter than at Saturn. Thus, for example, in the Jupiter models constructed by Cowley and Bunce (2001), the peak azimuth-integrated currents flowing in each hemisphere are of the order of $\sim 100 \mathrm{MA}$, as opposed to the $\sim 6 \mathrm{MA}$ found here for Saturn. As in the Saturn models, the jovian currents then flow out of the ionosphere into the magnetosphere in the region equatorward of the peak, where the plasma approaches rigid corotation. However, as a consequence of the distended nature of the middle magnetosphere field lines at Jupiter, this region has a much narrower latitudinal extent at Jupiter than at Saturn. The latitudinal width at Jupiter is $\sim 1^{\circ}$, compared with $\sim 5^{\circ}-10^{\circ}$ estimated here for Saturn. The field-aligned current density is thus increased at Jupiter by the same factor. Overall, therefore, the peak upward field-aligned current densities at Jupiter are about two orders of magnitude larger than those at Saturn, $\sim 1 \mu \mathrm{A} \mathrm{m}^{-2}$ at Jupiter compared with $\sim 10 \mathrm{nA} \mathrm{m}^{-2}$ at Saturn. The jovian currents are then in excess of the limiting currents for fieldaligned acceleration $\left(\sim 10 \mathrm{nA} \mathrm{m}^{-2}\right)$ by about two orders of magnitude. This leads to the development of field-aligned voltages of $\sim 100 \mathrm{kV}$, which amplify the precipitating electron energy flux from a diffuse background of $\sim 0.1 \mathrm{~mW} \mathrm{~m}^{-2}$ ( $\sim 1 \mathrm{kR}$ of UV emission) to $\sim 100 \mathrm{~mW} \mathrm{~m}^{-2}$ ( $\sim 1 \mathrm{MR}$ of UV emission). The latter precipitation is thus suggested as the origin of the 'main oval' auroras at Jupiter (Bunce and Cowley, 2001; Cowley and Bunce, 2001). By comparison, the peak currents at Saturn are significantly smaller than the limiting current $\left(\sim 70 \mathrm{nA} \mathrm{m}^{-2}\right)$, such that significant fieldaligned voltages are not expected to be produced, and the precipitating electron energy flux is not elevated above the diffuse background of $\sim 0.02 \mathrm{~mW} \mathrm{~m}^{-2}(\sim 0.2 \mathrm{kR}$ of UV emission).
The above conclusions apply to the system of field-aligned currents associated with the large-scale variations of the plasma angular velocity, in which the latter falls steadily from near-rigid corotation at small equatorial distances from the planet, to some fraction of rigid corotation in the outer regions. However, the Voyager data also suggest the occasional presence of smaller-scale 'dips' in the plasma angular velocity relative to the large-scale variation, by $\left(\Delta \omega / \Omega_{S}\right) \approx$ $0.2-0.3$, occurring in regions of radial extent $\sim 1-2 R_{S}$ in the equatorial plane. During the inbound Voyager-1 pass, for example, one such feature was observed centred near $\sim 10 R_{S}$, just outside the orbit of Rhea, while another was observed centred near $\sim 7 R_{S}$, just outside the orbit of Dione. Here, we have assumed that these observations correspond to spacecraft traversals of radially restricted, but azimuthally-extended flow structures. With the parameters employed here, such angular velocity features produce enhancements in the equatorward-directed ionospheric Pedersen currents which carry additional azimuth-integrated currents of $\sim 6 \mathrm{MA}$, comparable to the large-scale currents, in regions having latitudinal widths of $\sim 1^{\circ}-2^{\circ}$. Current continuity then requires the presence of a corresponding bipolar pattern of field-aligned currents, with downward currents flowing in the poleward region and upward currents flowing immediately equatorward. However, because the spatial scale of the current variation is an order of magnitude less than that of the large-scale currents, while the total currents are comparable, the field-aligned current densities are an order of magnitude larger than those of the large-scale system discussed above, peaking at upward and downward values of $\sim 100 \mathrm{nA} \mathrm{m}^{-2}$. Such values do modestly exceed our estimated limiting current requiring field-aligned electron acceleration, such that modest auroral signatures appear feasible under favourable circumstances. Accelerating voltages of the order of a few hundred volts are anticipated, yielding enhanced precipitating energy fluxes of $\sim 0.1 \mathrm{~mW} \mathrm{~m}^{-2}$, corresponding to $\sim 1 \mathrm{kR}$ of auroral emission. The anticipated auroras take the form of approximately axisymmetric rings of emission about each pole (or partial rings depending on their azimuthal extent), $\sim 0.25^{\circ}$ wide, somewhat dimmer in the south than in the north, and located at co-latitudes in the range $\sim 17^{\circ}$ and $\sim 20^{\circ}$ in the north and $\sim 19^{\circ}$ and $\sim 22^{\circ}$ in the south. No reports of such emissions have yet appeared in the literature, but equally it is unclear whether any of the investigations reported to date would have had the sensitivity to detect them had they actually been present. In the magnetosphere, correlated enhancements in the azimuthal perturbation field are expected to accompany the reductions in the angular velocity associated with these effects (see Fig. 1), though we may comment that none are very evident in the Voyager-1 magnetic data shown in the companion paper. Further study of these phenomena with future Cassini data is thus warranted.

Acknowledgements. We would like to thank Joe Mafi at the Planetary Data Center, UCLA, for supplying us with plasma and magnetic field data from the Voyager Saturn flybys. We would also 
like to thank John Clarke (Boston University) and Denis Grodent (Université de Liège) for helpful comments on the relation between precipitated electron energy flux and auroral UV emission intensity. EJB was supported during the course of this study by PPARC Grant PPA/G/O/1999/00181 and SWHC by PPARC Senior Fellowship PPA/N/S/2000/00197.

Topical Editor T. Pulkkinen thanks two referees for their help in evaluating this paper.

\section{References}

Atreya, S. K., Waite, Jr., J. H., Donahue, T. M., Nagy, A. S., and McConnell, J. C.: Theory, measurements and model of the upper atmosphere and ionosphere of Saturn, edited by T. Gehrels and Matthews, M. S., p. 239, Univ. Arizona Press, Tucson, Arizona, USA, 1984.

Behannon, K. W., Lepping, R. P., and Ness, N. F.: Structure and dynamics of Saturn's outer magnetosphere and boundary regions, J. Geophys. Res., 88, 8791, 1983.

Broadfoot, A. L., Sandel, B. R., Shemansky, D. E., Holberg, J. B., Smith, G. R., Strobel, D. F., McConnell, J. C., Kumar, S., Hunten, D. M., Atreya, S. K., Donahue, T. M., Moos, H. W., Bertaux, J. L., Blamont, J. E., Pomphrey, R. B., and Linik, S.: Extreme ultraviolet observations from Voyager 1 encounter with Saturn, Science, 212, 206, 1981.

Bunce, E. J. and Cowley, S. W. H.: Divergence of the equatorial current in the dawn sector of Jupiter's magnetosphere: analysis of Pioneer and Voyager magnetic field data, Planet. Space Sci., 49, 1089, 2001.

Bunce, E. J. and Cowley, S. W. H.: A note on the ring current in Saturn's magnetosphere: Comparison of magnetic data obtained during the Pioneer-11 and Voyager-1 and -2 flybys, Ann. Geophysicae, 21, 661, 2003.

Bunce, E. J., Cowley, S. W. H., and Wild, J. A.: Azimuthal magnetic fields in Saturn's magnetosphere: Effects associated with plasma sub-corotation and the magnetopause-tail current system, Ann. Geophysicae, in this issue, 2003.

Clarke, J. T., Moos, H. W., Atreya, S. K., and Lane A. L.: IUE detection of bursts of H Ly $\alpha$ emission from Saturn, Nature, 290, 226, 1981.

Cheng, A. F. and Waite, Jr., J. H.: Corotation lag of Saturn's magnetosphere: Global ionospheric conductivities revisited, J. Geophys. Res., 93, 4107, 1988.

Clarke, J. T., Ballester, G., Trauger, J., Ajello, J., Pryor, W., Tobiska, K., Connerney, J. E. P., Gladstone, G. R., Waite, Jr., J. H., Ben Jaffel, L., and Gérard J.-C.: Hubble Space Telescope imaging of Jupiter's UV aurora during the Galileo orbiter mission, J. Geophys. Res., 103, 20 217, 1998.

Connerney, J. E. P., Acuña, M. H., and Ness, N. F.: Modeling the jovian current sheet and inner magnetosphere, J. Geophys. Res. 86, 8370, 1981.

Connerney, J. E. P., Ness, N. F., and Acuña, M. H.: Zonal harmonic model of Saturn's magnetic field from Voyager-1 and -2 observations, Nature, 298, 44, 1982.

Connerney, J. E. P., Acuña, M. H., and Ness, N. F.: Currents in Saturn's magnetosphere, J. Geophys. Res., 88, 8779, 1983.

Connerney, J. E. P., Acuña, M. H., and Ness, N. F.: The $Z_{3}$ model of Saturn's magnetic field and the Pioneer 11 vector helium magnetometer observations, J. Geophys. Res., 89, 7541, 1984.

Cowley, S. W. H. and Bunce E. J.: Origin of the main auroral oval in Jupiter's coupled magnetosphere-ionosphere system, Planet.
Space Sci., 49, 1067, 2001.

Davis, Jr., L. and Smith, E. J.: A model of Saturn's magnetic field based on all available data, J. Geophys. Res., 95, 15 257, 1990.

Desch, M. D.: Evidence for solar wind control of Saturn radio emission, J. Geophys. Res., 87, 4549, 1982.

Desch, M. D. and Rucker H. O.: The relationship between Saturn kilometric radiation and the solar wind, J. Geophys. Res., 88, 8999, 1983.

Edwards, T. M., Bunce, E. J., and Cowley, S. W. H.: A note on the vector potential of Connerney et al.'s model of the equatorial current sheet in Jupiter's magnetosphere, Planet. Space Sci., 49, $1115,2001$.

Espinosa, S. A. and Dougherty, M. K.: Periodic perturbations in Saturn's magnetic field, Geophys. Res. Lett., 27, 2785, 2000.

Frank, L. A., Burek, B. G., Ackerson, K. L., Wolfe, J. H., and Mihalov, J. D.: Plasmas in Saturn's magnetosphere, J. Geophys. Res., 85, 5695, 1980.

Gérard, J.-C., Dols, V., Prangé, R., and Paresce, F.: The morphology of the north Jovian ultraviolet aurora observed with the Hubble Space Telescope, Planet. Space Sci., 42, 905, 1994.

Gérard, J.-C., Dols, V., Grodent, D., Waite, J. H., Gladstone, G. R., and Prangé, R.: Simultaneous observations of the saturnian aurora and polar haze with the HST/FOC, Geophys. Res. Lett., 22, 2685, 1995.

Grodent, G., Clarke, J. T., Kim, J., Waite, Jr., J. H., and Cowley, S. W. H.: Jupiter's main auroral oval observed with HST-STIS, J. Geophys. Res., in press, 2003.

Gurnett, D. A., Kurth, W. S., and Scarf, F. W.: Plasma waves near Saturn: Initial results from Voyager 1, Science, 212, 235, 1981.

Hill, T. W.: Inertial limit on corotation, J. Geophys. Res., 84, 6554, 1979.

Hill, T. W.: The jovian auroral oval, J. Geophys. Res., 106, 8101, 2001.

Huang, T. S. and Hill T. W.: Corotation lag of the jovian atmosphere, ionosphere and magnetosphere, J. Geophys. Res., 94, 3761, 1989.

Judge, D. L., Wu, F. M., and Carlson, R. W.: Ultraviolet photometer observations of the Saturnian system, Science, 207, 431, 1980.

Kaiser, M. L. and Desch M. D.: Saturnian kilometric radiation: Source location, J. Geophys. Res., 87, 4555, 1982.

Kaiser, M. L., Desch, M. D., Kurth, W. S., Lecacheux, A., Genova, F., Pederson, B. M., and Evans, D. R.: Saturn as a radio source, in Saturn, edited by Gehrels, T. and Matthews, M. S., p. 378, Univ. Arizona Press, Tucson, Arizona, USA, 1984.

Knight, S.: Parallel electric fields, Planet. Space Sci., 21, 741, 1973.

Lecacheux, A. and Genova, F.: Source location of Saturn kilometric radio emission, J. Geophys. Res., 88, 8993, 1983.

Lundin, R. and Sandahl, I.: Some characteristics of the parallel electric field acceleration of electrons over discrete auroral arcs as observed from two rocket flights, in: Symposium on European Rocket Research, ESA SP-135, ESA, Noordwijk, The Netherlands, p. 125, 1978.

Maurice, S. and Engel, I. M.: Idealized Saturn magnetosphere shape and field, J. Geophys. Res., 100, 17 143, 1995.

McGrath, M. A. and Clarke, J. T.: H I Lyman alpha emission from Saturn (1980-1990), J. Geophys. Res., 97, 13 691, 1992.

Moses, J. I. and Bass, S. F.: The effects of external material on the chemistry and structure of Saturn's ionosphere, J. Geophys. Res., 105, 7013, 2000.

Ness, N. F., Acuña, M. H., Lepping, R. P., Connerney, J. E. P., Behannon, K. W., Burlaga, L. F., and Neubauer, F. M.: Magnetic field studies by Voyager 1: Preliminary results at Saturn, Science, 
212, 211, 1981

Prangé, R., Rego, D., Pallier, L., Connerney, J. E. P., Zarka, P., and Queinnec, J.: Detailed study of FUV jovian auroral features with the post-COSTAR HST faint object camera, J. Geophys. Res., 103, $20195,1998$.

Richardson, J. D.: Thermal ions at Saturn: Plasma parameters and implications, J. Geophys. Res., 91, 1381, 1986.

Richardson, J. D.: A new model for plasma transport and chemistry at Saturn, J. Geophys. Res., 97, 13 705, 1992.

Richardson, J. D.: An extended plasma model for Saturn, Geophys. Res. Lett., 22, 1177, 1995.

Richardson, J. D. and Sittler, Jr., E. C.: A plasma density model for Saturn based on Voyager observations, J. Geophys. Res., 95, $12019,1990$.

Richardson, J. D., Eviatar, A., McGrath, M. A., and Vasyliunas, V. M.: OH in Saturn's magnetosphere: Observations and theory, J. Geophys. Res., 103, 20 245, 1998.

Sandel, B. R. and Broadfoot, A. L.: Morphology of Saturn's aurora, Nature, 292, 679, 1981.

Sandel, B. R., Shemansky, D. E., Broadfoot, A. L., Holberg, J. B., Smith, G. R., McConnell, J. C., Strobel, D. F., Atreya, S. K., Donahue, T. M., Moos, H. W., Hunten, D. M., Pomphrey, R. B., and Linik, S.: Extreme ultraviolet observations from the Voyager 2 encounter with Saturn, Science, 215, 548, 1982.

Satoh, T., Connerney, J. E. P., and Baron, R. L.: Emission source model of Jupiter's $\mathrm{H}_{3}^{+}$aurorae: A generalized inverse analysis of images, Icarus, 122, 1, 1996.

Shemansky, D. E. and Ajello, J. M.: The Saturn spectrum in the EUV: Electron excited hydrogen, J. Geophys. Res., 88, 459, 1983.

Sittler, Jr., E. C., Ogilvie, K. W., and Scudder, J. D.: Survey of lowenergy plasma electrons in Saturn's magnetosphere: Voyagers-1 and -2, J. Geophys. Res., 88, 8847, 1983.

Southwood, D. J. and Kivelson, M. G.: A new perspective concerning the influence of the solar wind on Jupiter, J. Geophys. Res. 106, 6123, 2001.

Trauger, J. T., Clarke, J. T., Ballester, G. E., Evans, R. W., Burrows, C. J., Crisp, D., Gallagher, J. S., III, Griffiths, R. E., Hester, J. J., Hoessel, J. G., Holtzman, J. A., Krist, J. E., Mould, J. R., Sahai, R., Scowen, P. A., Stapelfeldt, K. R., and Watson, A. M.: Saturn's hydrogen aurora: Wide field and planetary camera 2 imaging from the Hubble Space Telescope, J. Geophys. Res., 103, $20237,1998$.

Vasavada, A. R., Bouchez, A. H., Ingersoll, A. P., Little, B., Anger, C. D., and the Galileo SSI Team: Jupiter's visible aurora and Io footprint, J. Geophys. Res., 104, 27 133, 1999.

Vasyliunas, V.M.: Plasma distribution and flow, in: Physics of the Jovian Magnetosphere, edited by Dessler, A. J., p. 395, Cambridge Univ. Press, Cambridge, UK, 1983. 